
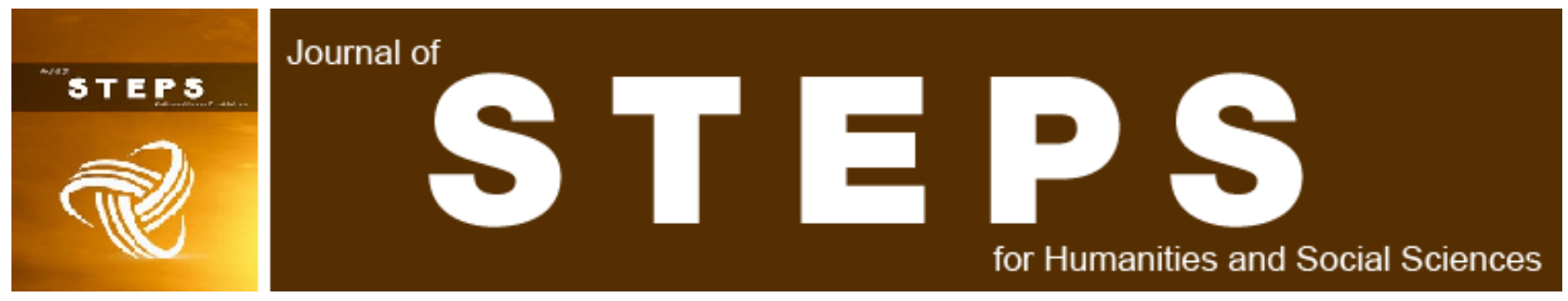

Volume 1 | Issue 1

Article 8

\title{
Ecological architecture and its impact on human behavior and human health
}

Rabab Jassim

University of Technology, Iraq, ae.20.46@grad.uotechnology.edu.iq

Sabeeh Farhan

Wasit University, Iraq

Shamael Al-Dabbagh

University of Technology, Iraq

Zahraa Shamam

University of Technology, Iraq

Follow this and additional works at: https://www.steps-journal.com/jshss

Part of the Arts and Humanities Commons

\section{Recommended Citation}

Jassim, Rabab; Farhan, Sabeeh; Al-Dabbagh, Shamael; and Shamam, Zahraa (2022) "Ecological architecture and its impact on human behavior and human health," Journal of STEPS for Humanities and Social Sciences: Vol. 1 : Iss. 1 , Article 8.

Available at: https://doi.org/10.55384/2790-4237.1002

This Original Study is brought to you for free and open access by Journal of STEPS for Humanities and Social Sciences (STEPS). It has been accepted for inclusion in Journal of STEPS for Humanities and Social Sciences by an authorized editor of Journal of STEPS for Humanities and Social Sciences (STEPS). 


\section{العمارة الأيكولوجية وتأثيرها في السلوك البشري وصحة الانسان}

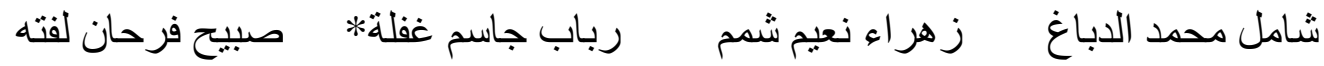

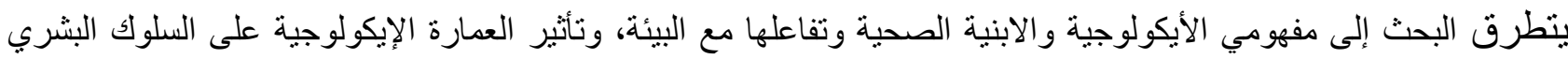

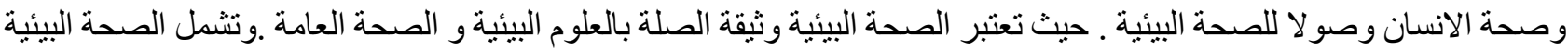

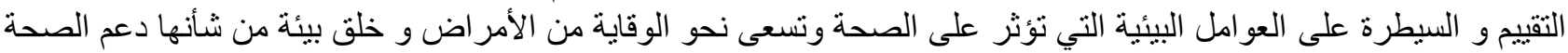

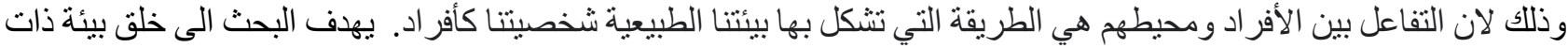

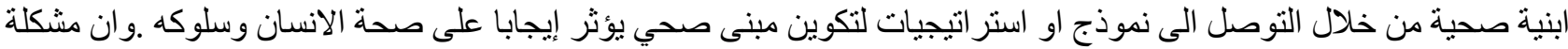

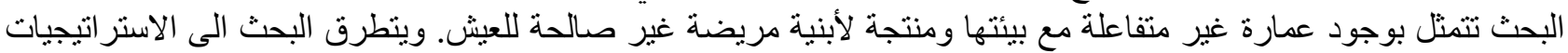
التي توفر اعتدال حراري وتقلل التلوث وتوفر الطاقة وذلك من خلال المدخلات والمخرجات ما بين المبنى والبيئة. أثنارت

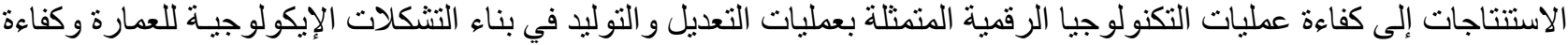

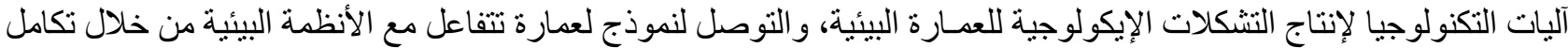

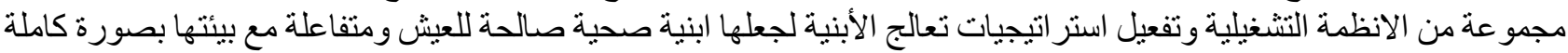

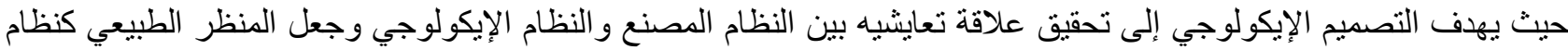

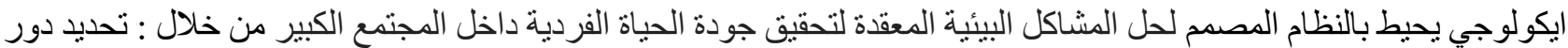

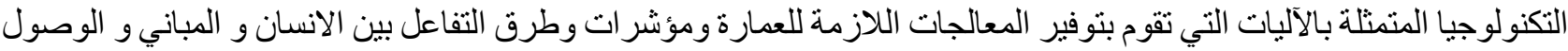

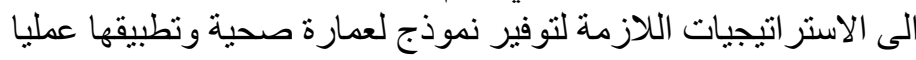

كلمات مفتاحية: الايكولوجي،علم النفس البيئي ،الأبنية الصحية، الأبنية المريضة، صحة الانسان، السلوك الإنساني، الأيكولوجية، التكييف البئئي. 


\section{Ecological architecture and its impact on human behavior and human health}

Shamael Mohammed Al-Dabbagh, Architecture Department, University of Technology, Iraq.

Zahraa Naeem Shamam, Architecture Department, University of Technology, Iraq.

Rabab Jassim Ghaflah, Architecture Department, University of Technology, Iraq.

Sabeeh Lafta Farhan, Architecture Department, University of Wasit, Iraq.

\section{Abstract}

The research deals with the concepts of ecological and healthy buildings and their interaction with the environment, and the impact of ecological architecture on human behavior and human health, leading to environmental health. Where environmental health is closely related to environmental sciences and public health. Environmental health includes the assessment and control of environmental factors that affect health and seeks to prevent diseases and create an environment that supports health because the interaction between individuals and their surroundings is the way in which our natural environment is formed Our character as individuals. The research aims to create an environment with healthy buildings by finding a model or strategies for creating a healthy building that positively affects human health and behavior. The research deals with strategies that provide thermal moderation, reduce pollution and save energy, through the inputs and outputs between the building and the environment. The conclusions indicated the efficiency of digital technology processes represented in the processes of modification and generation in building the ecological formations of architecture and the efficiency of technology mechanisms to produce the ecological formations of environmental architecture, and reaching a model for architecture that interacts with environmental systems through the integration of a set of operational systems and activating strategies that address buildings to make them healthy, livable and interactive buildings Its environment completely, as ecological design aims to achieve a symbiotic relationship between the factory system and the ecosystem and make the landscape as an ecosystem surrounding the system designed to solve complex environmental problems to achieve individual quality of life within the large community through: Defining the role of technology represented by mechanisms that provide the necessary treatments for architecture and indicators And ways of interaction between humans and buildings, and access to the necessary strategies to provide a model for healthy architecture and apply them in practice.

Keywords: Ecology, environmental psychology, healthy buildings, sick buildings, human health, human behavior, ecology, environmental conditioning. 
تعود جذور الحركة البيئية الى القرن التاسع عشر بعد التطورات التي حصلت في العلوم الطبيعية كانت من

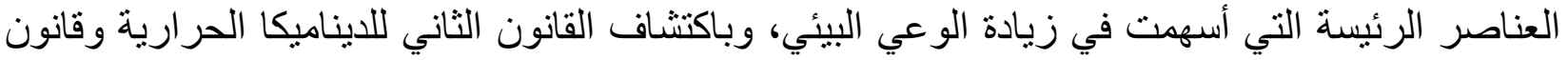

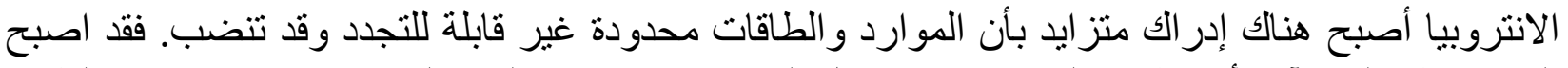

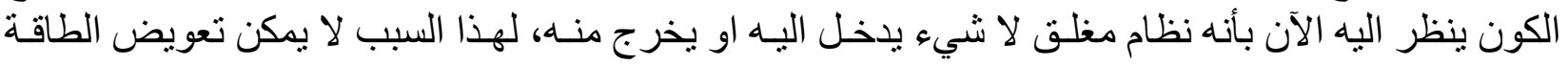

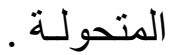

بعد ذلك ظهرت توجهات جديدة بدأت في التفكير في الكيفية التي سيجري بها تنظيم المجتمع وفي علاقة الإنسان

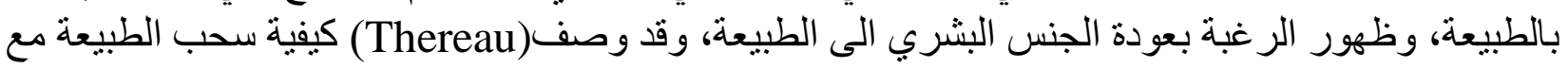

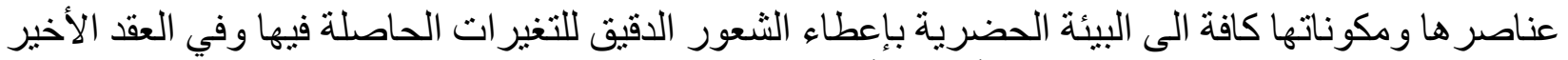

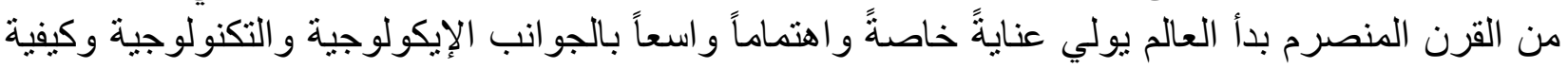
استخلاله لتحقيق مباني او بيئات صحية لتحقيق مباني تفاعليه مع الفرد الذي الذي تهيمن الأحاسيس على ردئ ردود افعاله

اتجاه المباني[1].

\section{المحور الأول: الاطار المعرفي}

مفهوم الإيكولوجي : العلم الذي يختص بالعلاقات بين الكائنات الحية و المحيط الفيزيائي ، أو البيئة المحيطة بها

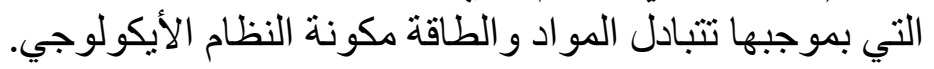

مصطلح Ecology مشتق من كلمة Okologi وتعني علاقة الكائن الحي مع المكونات العضوية و اللاعضوية

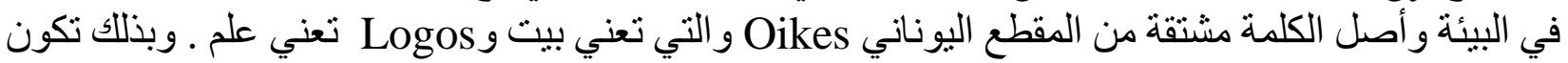

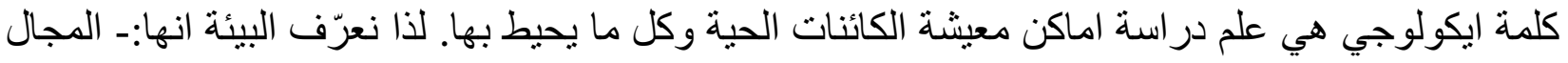
التي تحدث فيه الاثارة و التفاعل لكل وحدة حية وهي كل ما يحيط بالإنسان من طبيعة و وجتمعات بشرية ونظة

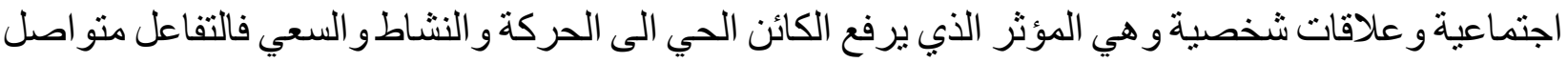

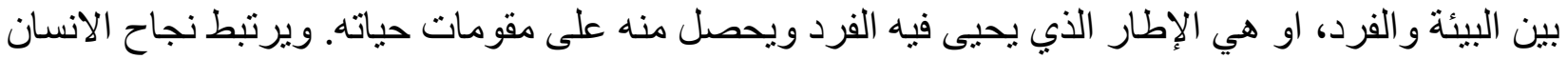

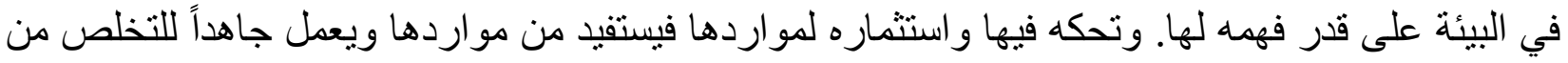
الملوثات التي فيها والتي تؤثر تأثير ات ذات ذات ابعاد مختلفة عليه.

الأبنيةة المريضة :- هي الأبنية التي تعتمد على الأنظمة الصناعية بشكل تام وتنقطع عن بيئتها الأبنية الصحية :- هي الأبنية المتفاعلة مع بيئتها وتأخذ بنضر الاعتبار الخصائص المناخية والحدود الطبيعية لموقع المشروع و مدى تأثثر ها على النظام المصدم و عمله بدلا من التركيز على المبنى فقط. التكنولوجيا المستدامة :-هي الفعالية أو التقنية التي تحقق الاستدامة بإحدى طر ائق تحوير الموارد إلى مصنعات

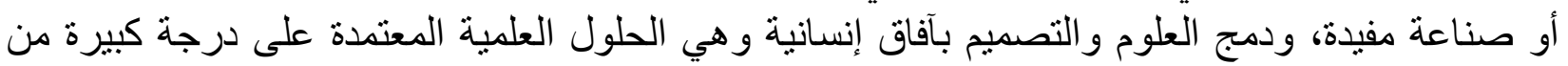
العقلانية و المو ائمة الطبيعية، وديجة إدومة

التكيف البيئي :- هو تفسير لتفاعل الإنسان مع مؤثرات البيئة الطبيعية و انعكاسها على المكونات الثكلية للمدينة

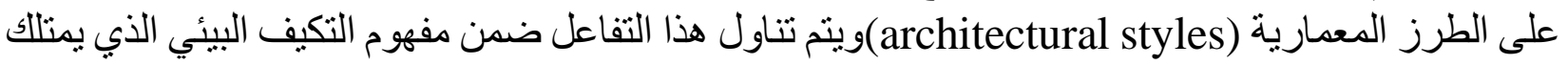
خاصية ثنائية تعمل بديناميكية مستمرة وبمستو المعات متعددة وفي ذلك صفات ثابتة ومتغيرة.

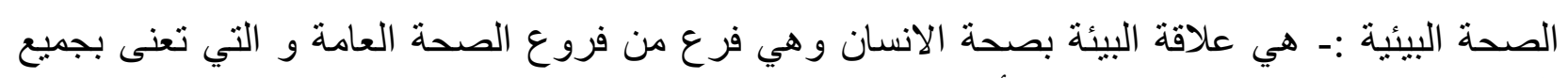

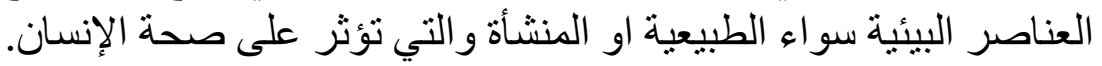

خدمات الصحة البيئية وفقا لمنظمة الصحة العالمية على انها: الخدمات التي تطبق سياسات الصحة البيئية من خلال أنشطة الرصد و المر اقبة. كما أنها تنفذ هذا الدور من خلال تعزيز وتحسين المعالئ المعايير البيئية وتشجيع 
استخدام تقنيات وسلوكيات صحية وصديقة للبيئة. ولهذه الخدمات دور ا رائدا في تطوير و اقتر اح مجالات جديدة[2]. كما موضح في الثكل رقم 1.

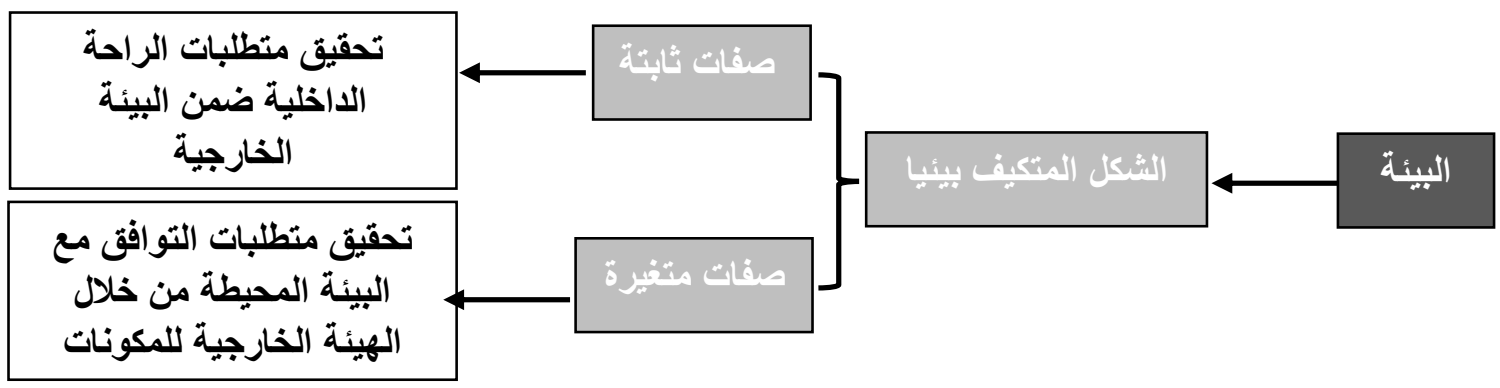

شكل ـ1.الصفات الثنابتة والمتغيرة للثكل المتيف بيئيا

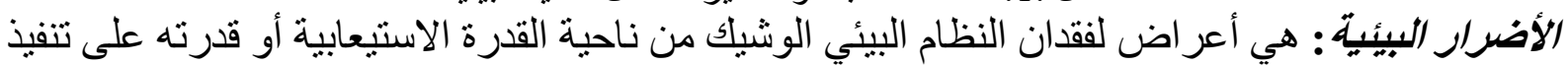

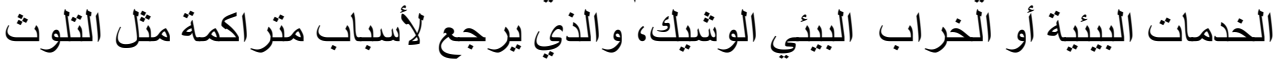

التصميم الإيكولوجي: هو التصميم الذي يعبر عنه بمصفوفة تمثل النظر إلى النظم التصميمية على هيئة

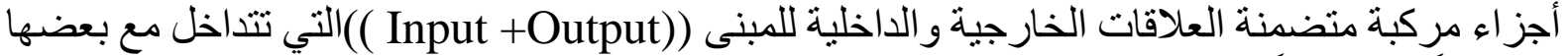

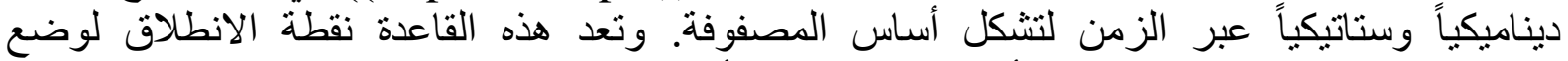
استر اتيجيات التصميم الإيكولوجي لأنماط مختلفة من الأبنية عن طريق معرفة النظم التشغيلية في البناية

خلال دورة حياتها.

التعريف الاجرائسي للتصديم الإيكوليوجي: هو التصميم المعتمد على البيئة و المتفاعل معها من خلال مجمو عه

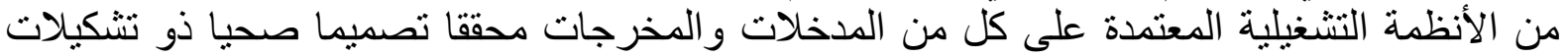

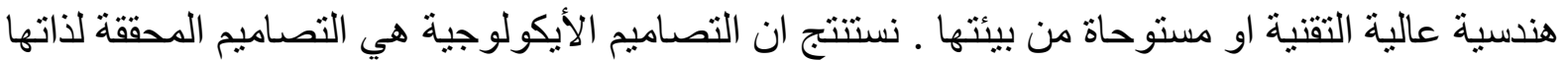
ولنظم الاستدامة البيئية فتتكامل انظمتها الطبيعية مع النمط الإنساني لإعطاء استمر ارية فريدة لصنع المكان

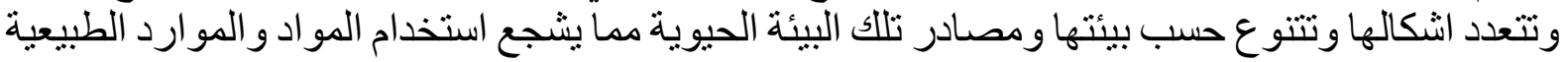
المحلية وتجنب استخدام الموارد و المو اد المضرة بالبيئة، والعناية بدورة المادة في الطبيعة، وتعزيز البنى التحنية، و الحفاظ على الابنية و اعادة تأهيلها [3].

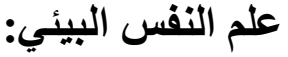

بدأ الاهتمام بعلم النفس البيئي مع ظهور نظريات الجشطالت في الاربعينيات من القرن

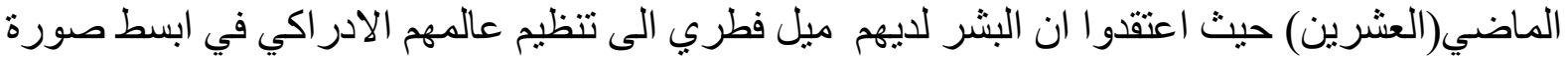

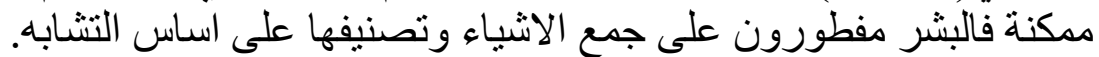

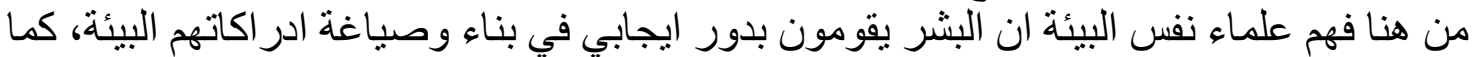

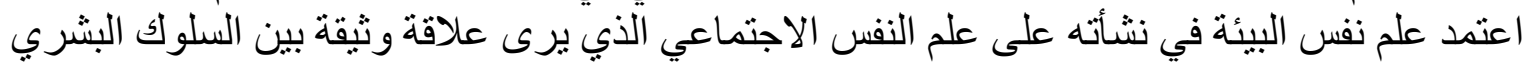
و البيئة الفيزيقية المحيطة بهاه.

- بدأ باركر ور ايت في اجر اهية در اسات جماعية في المدن عن تأثثير البيئة في سلوك الانسان.

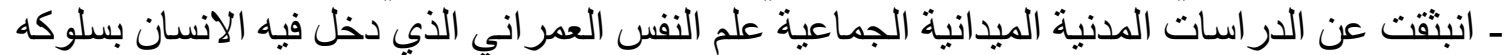

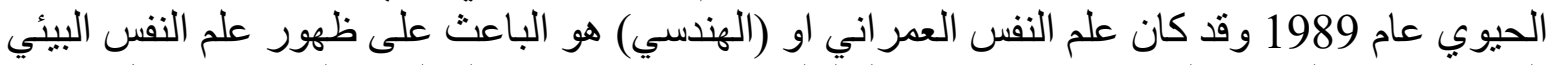

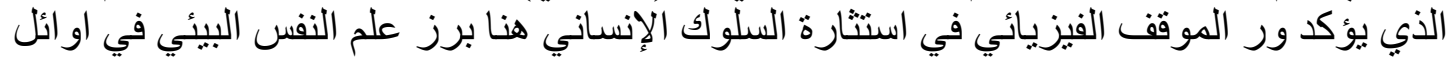

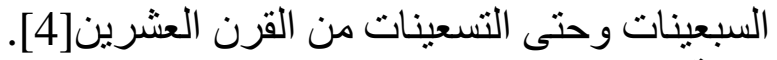
نظرية علم النفس الايكوليوجي :

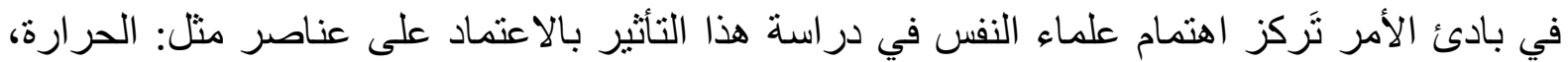
و الضوضاء، و التلوث و غير ها، وتأثثير ها على سلوك الأفر اد و الجماعات، ومن ثم ظهرت مفاهيم علم النفس

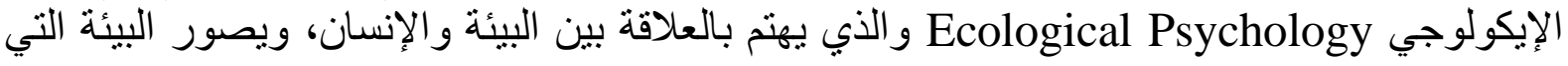


تؤثز على السلوك كنظام ديناميكي مركب من مجموعة من المكونات المادية والاجتماعية و هي المجال

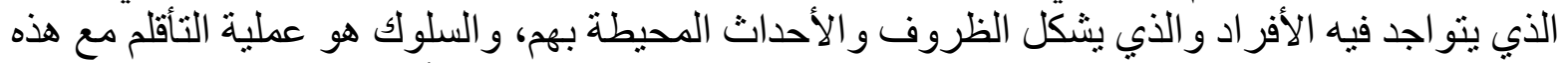

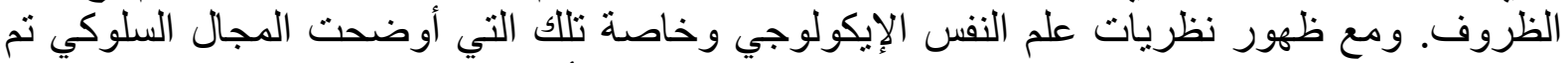

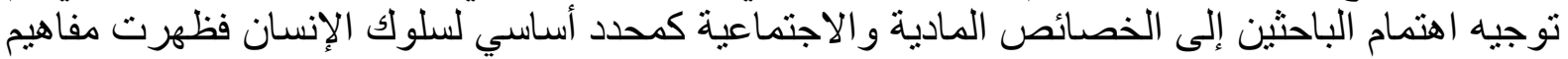

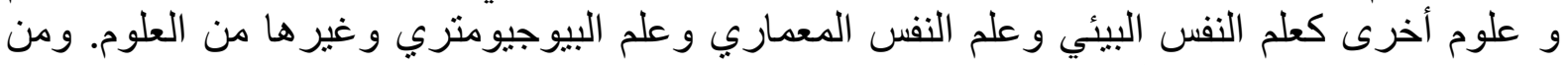
المو اضيع لتي تناو لتها هذه النظرية-: در اسة العلاقة بين المناخ (الهو اه، الحرار ةُ، والبة البرودة، و الأمطار ، و الجفاف و غير ها) وسلوك الإنسان مثل: ظروف التهوية وتجديد الهو اءو وتلوثه ومدى توفر النو افذ والمظلات و المناور و الهو ايات

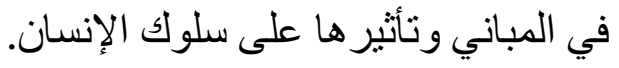

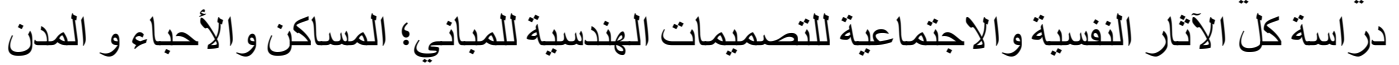

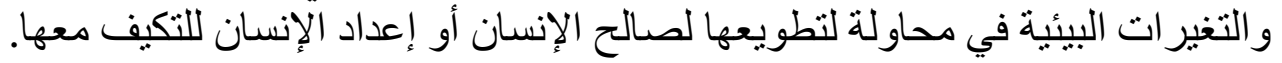

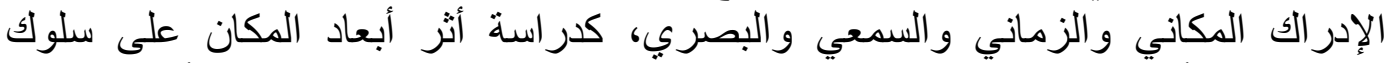

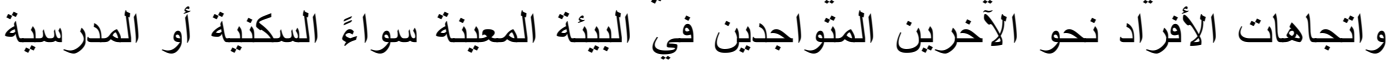
وغير ها. أنجات.

نستخلص إن هذه النظرية تؤكد على التفاعل بين الإنسان والبيئة المحيطه بها، وهو يشمل البيئة الطبيعية

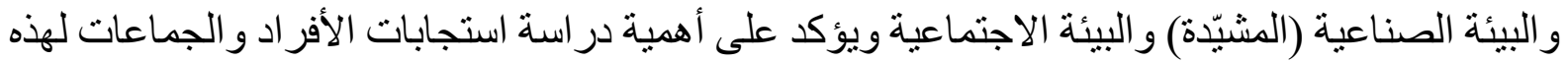

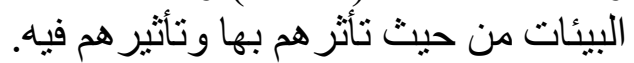

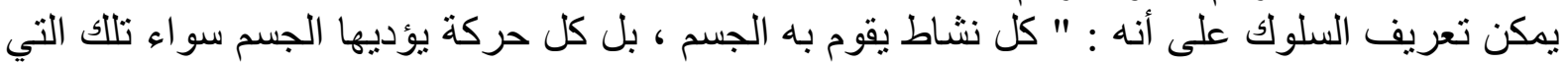

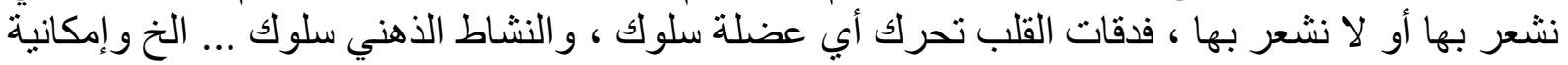

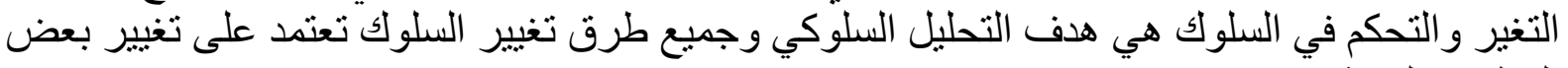

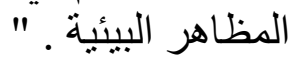

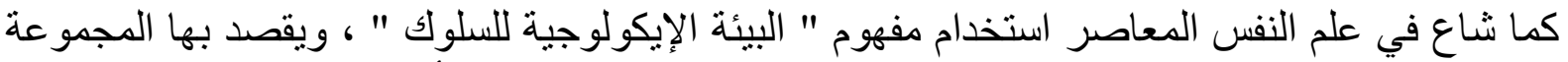
الكلية والمتكاملة من العو امل التي تثير السلوك وتنشطه ، كمدخل لتحديد ما لتأثير المتغير ات البيئية من دور

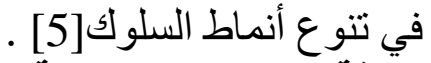
العلاقة بلين العدارة والماطبئة:

تتمثل العلاقة بين العمارة و البيئة بما يسمى بتقنية البناء ، وهو كل ما يتصل بالبناء من تطبيقات خلال

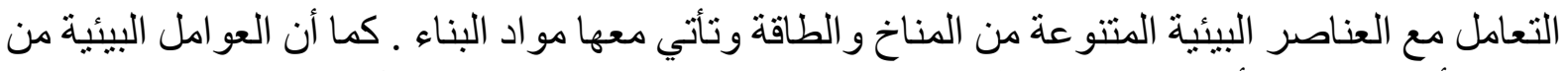

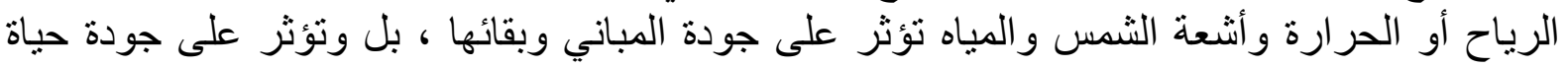

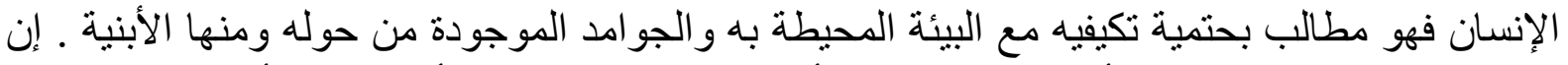
البناء عندما ينتهي يصبح جزءأ من البيئة كالثجر أو الحجر وبالتالي تتكون أنواع من الأبنية[6] :

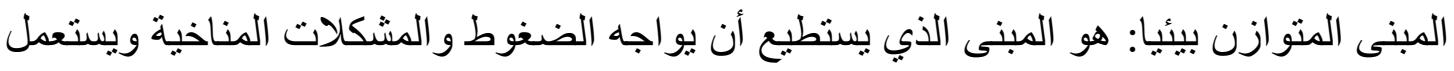

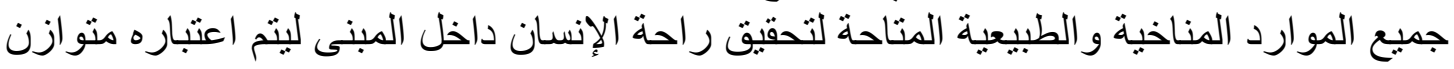

المباني المريضة: هي المباني التي تؤثر على نفسية الأفراد وتتحكم في السلوكيات التي يتصرفون بها في مختلف المو اقف.

وون بين خصائص المباني المريضة : ـ الإسر اف في استخدام الطاقة واستنز اف الموارد الطبيعية. ـ مو اد البناء ينبعث منها ما يسبب أضرارا بالغة الصحة الإنسان .

$$
\text { ـ ـاختيار موقع غير صحي للمبنى . }
$$




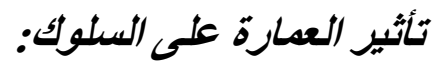

إن العمارة تؤثر على سلوكيات الإنسان ، فالتصميمات التي يضعها بنفسه والأعمال الإنشائية التي ينفذها

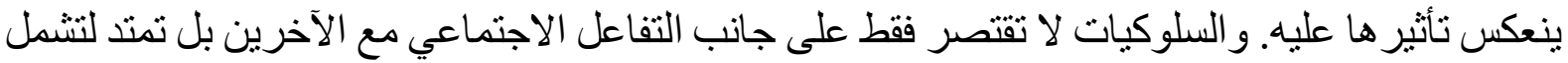

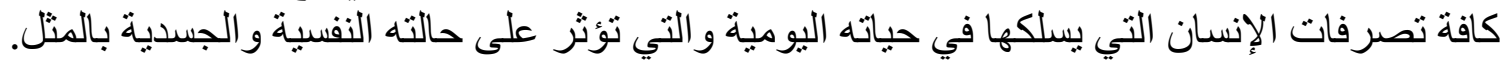

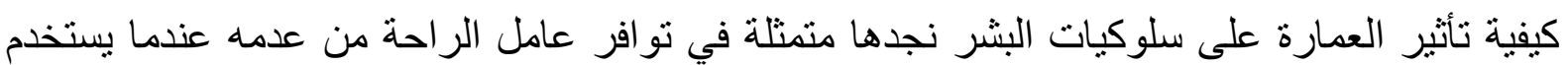

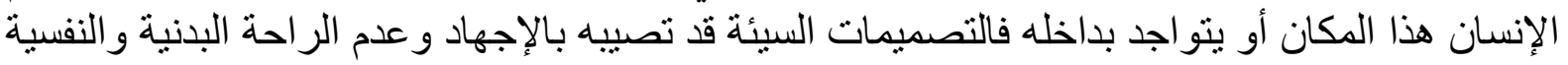

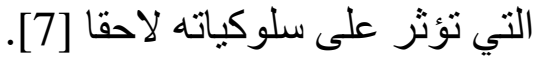

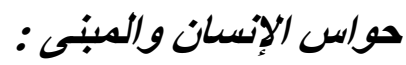

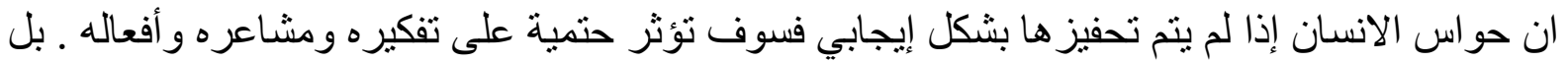

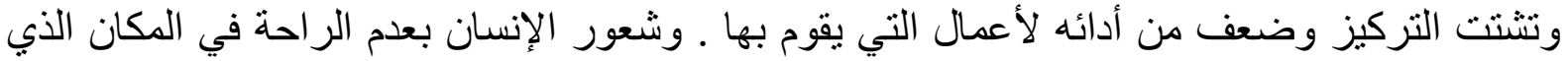

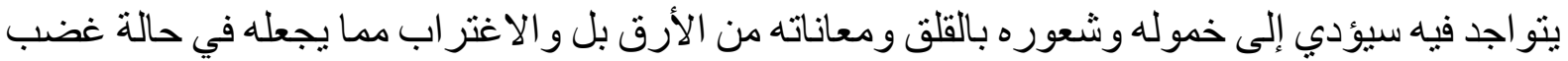

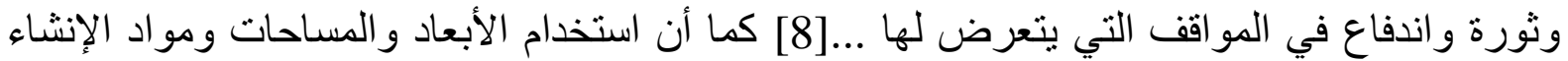

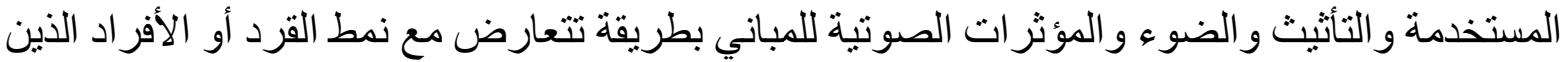

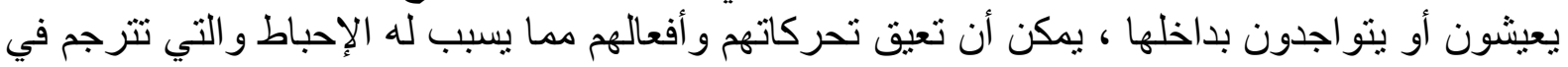

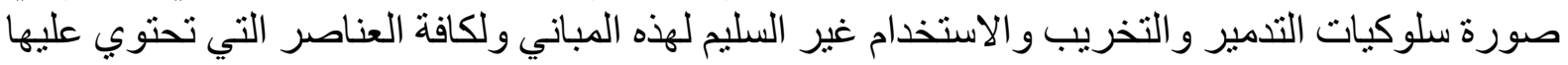

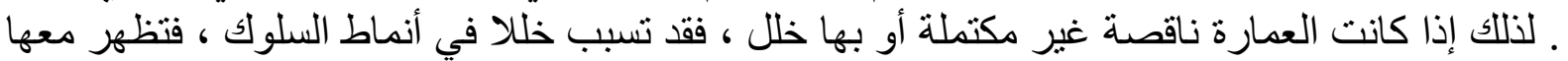

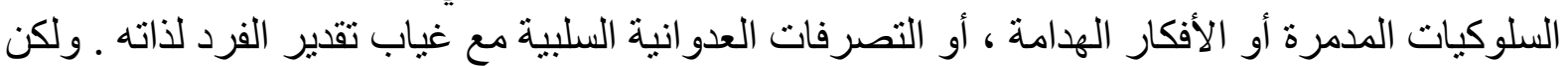

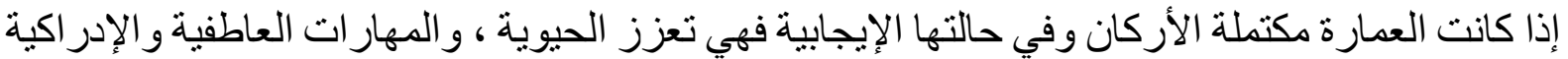

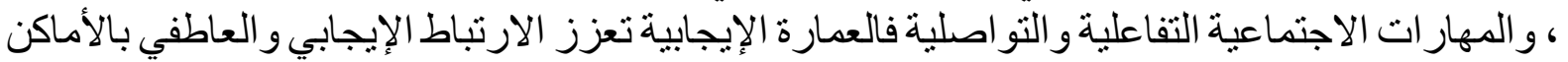

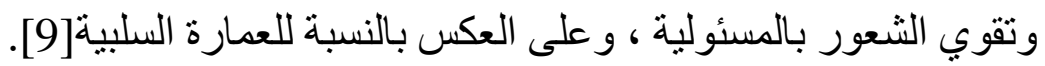

\section{التصميم الايكولوجي الاصلاحي ( Restorative environmental design ):}

اذ يثير هذا المصطلح و على وفق طروحات استاذ الايكولوجيا الاجتماعية ( Stephen R. Kellert ) من الن التئية

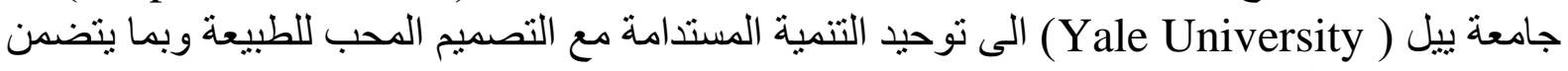

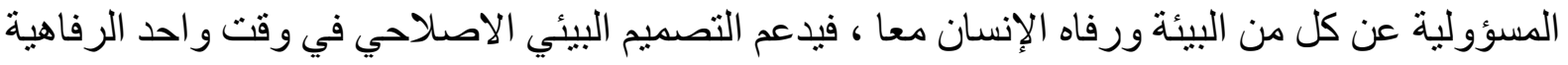

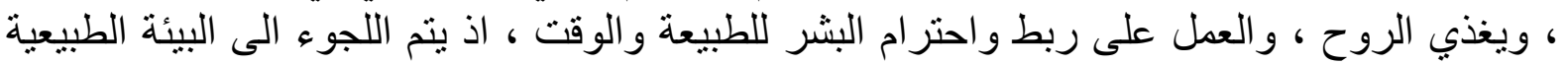

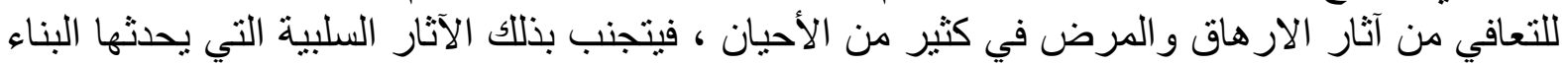

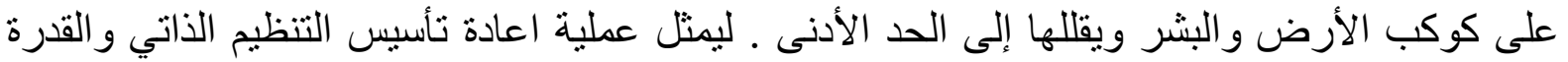

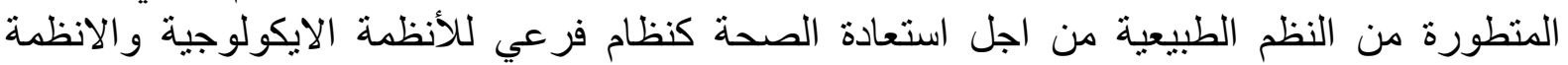

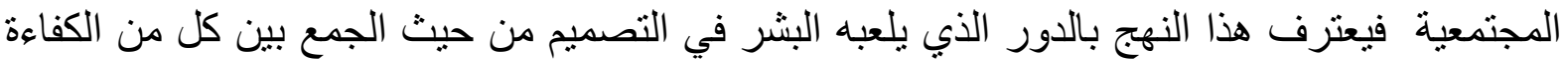

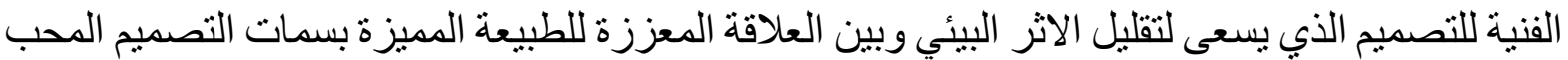

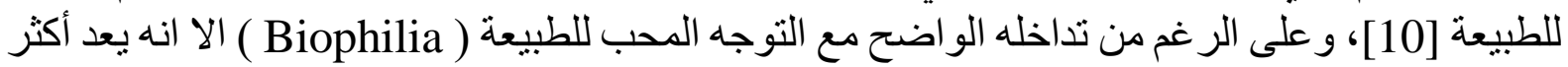

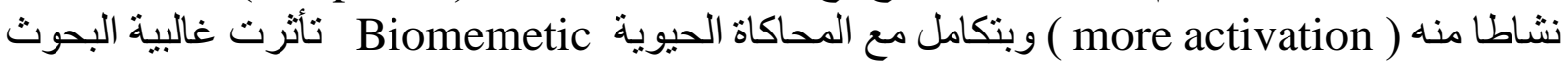

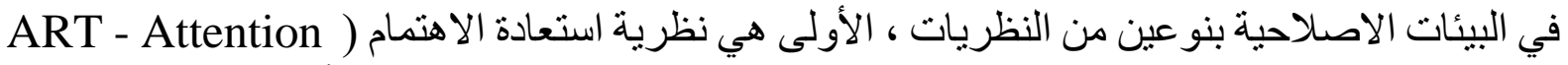
( Restoration Theory

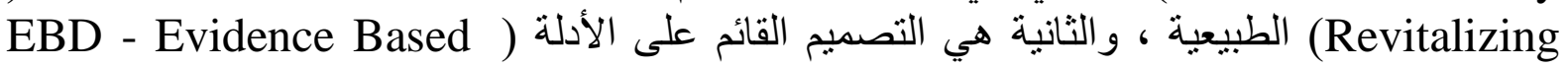
( Design

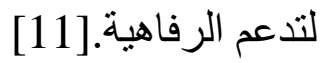




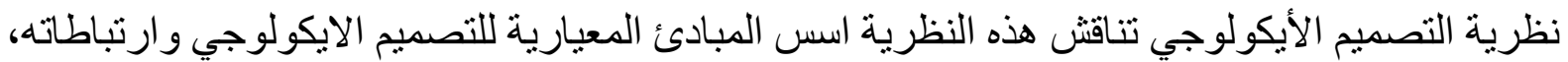

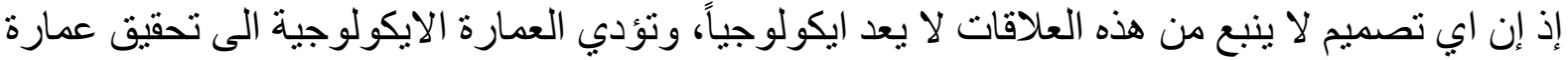

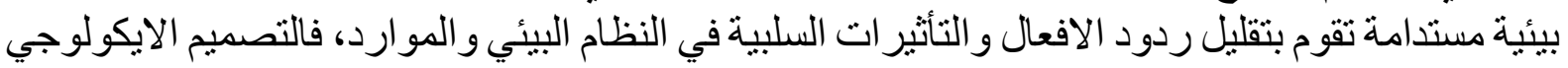

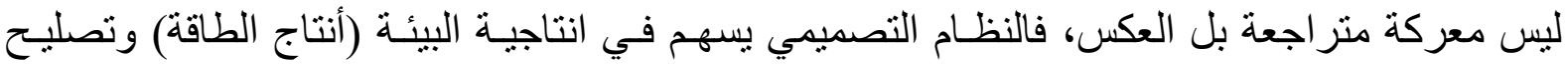
النظسام البيئي المتضـرر(Yeang,1999, p59) ويتحقق ذلك من خلال:

اولًا: البنبة النظرية للتصديم الايكولوجي النئي

إن العمل بالنظرية الايكولوجية يجب ان يعطي عددا من الافكار التي تتلخص بالاتي :

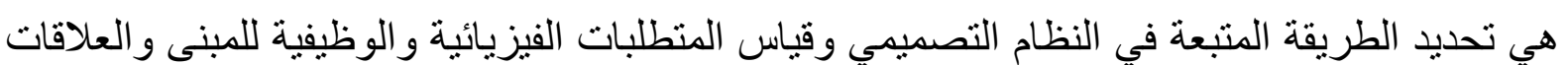
التر ابطية و علاقات النظام البيئي عن طريق الثكال الطاقة والموارد النئي المأخوذة من الطبيعة.

ثانيا: التوجهات الديناميكية للنظرية يؤكد المفهوم الديناميكي على التدفق الطبيعي للمو ارد ويشمل ذلك :-

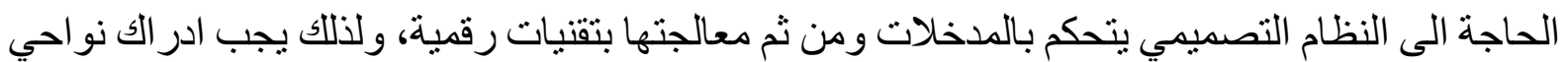

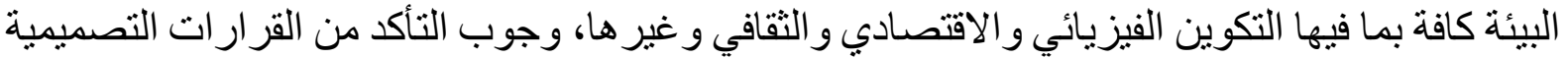

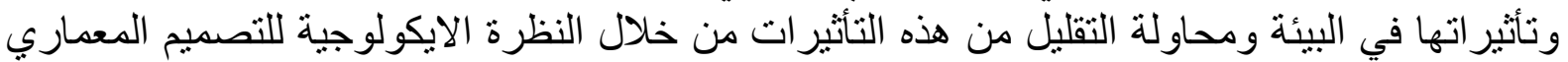

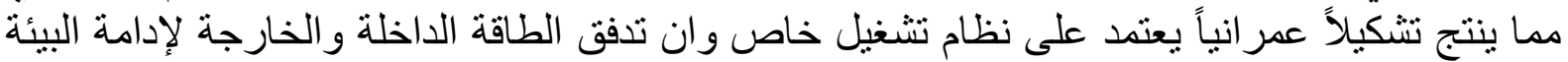
يجب ان تكون منوازنة [12].

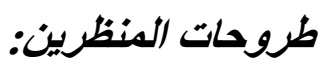

\section{• • طروحات (حسن فتحي، 1988)}

لقد وضحت الطروحات الأسس التي اعتمدت عليها العمارة التقليدية في الاستفادة القصوى من الطاقات الطبيعية، وإمكانية تطبيقها في العمارة الحديثة المحلية.

يشير الجزء الأول من الطروحات إلى دراسة العمارة التقليدية من نواح كثيرة منها ما ينعلق بالديناميكا

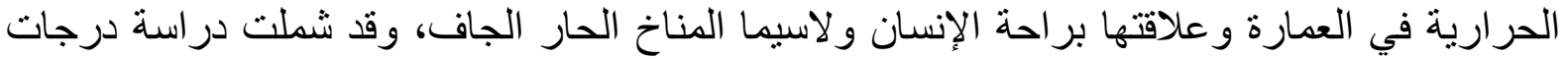

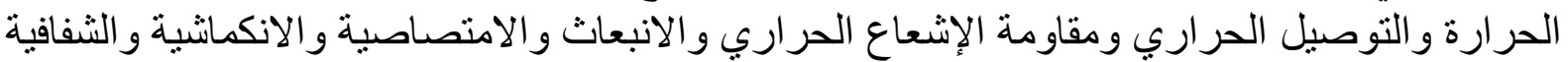

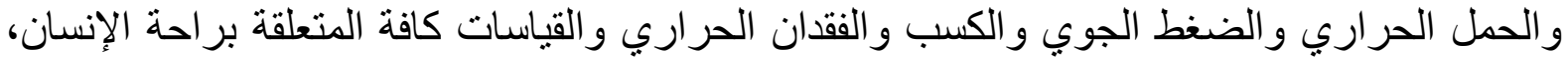

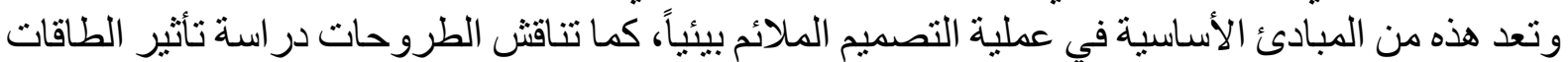

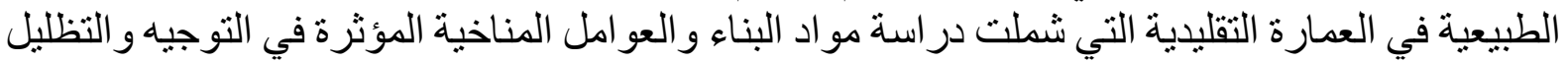

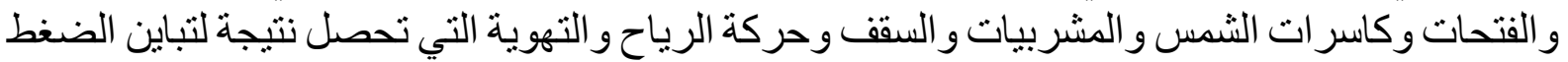
و وأثر الثشس في حركة الهواء[13].

ثم در اسة العو امل على مستوى البيت الواحد ذي الفناء الداخلي (الوسطي) و على مستوى تخطيط الددينة

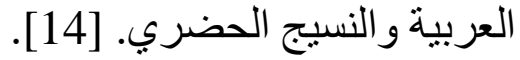

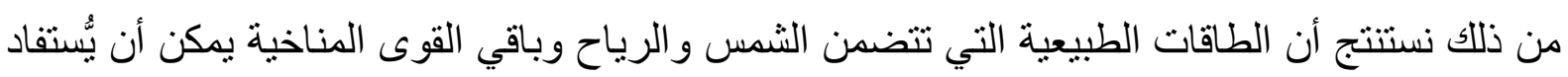

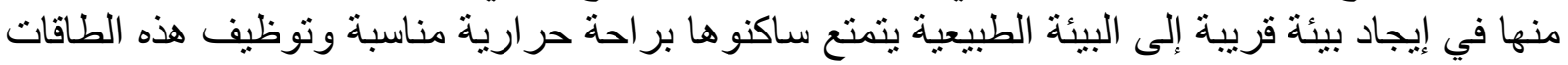

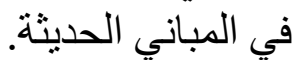


نستنتج من ذلك أن الطاقات الطبيعية التي تتضمن الثمس و الرياح وباقي القوى المناخية يمكن أن يُّتفاد

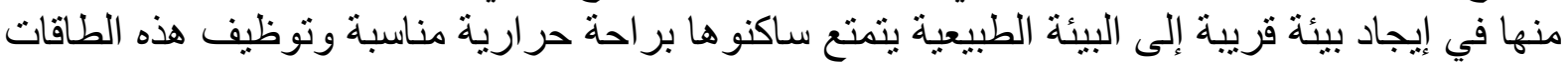

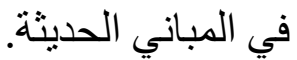

وييدو مما سبق أهمية النظم والتقنيات البسيطة كاستخدام الطاقات الطبيعية للتقليل من درجة الحرارة

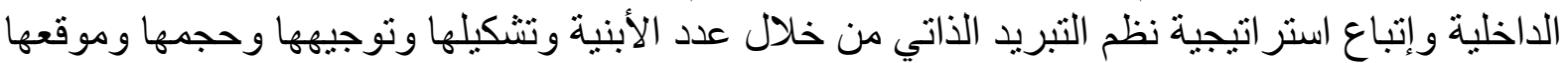

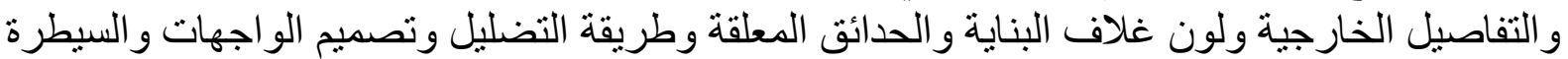

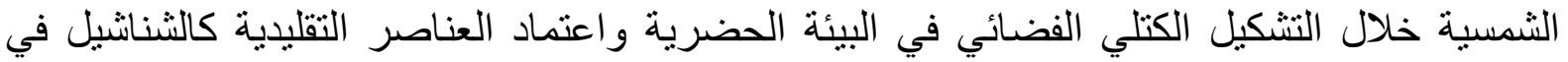

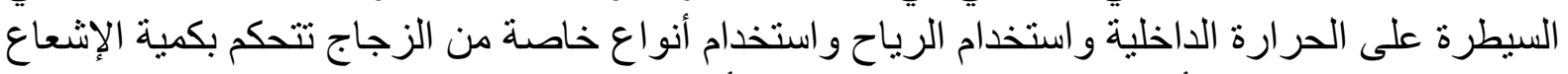

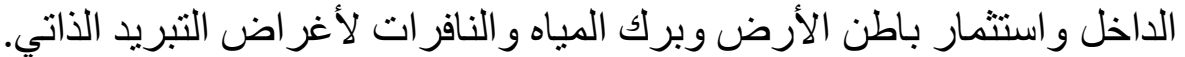

\section{(Yeang, 1995, 1999) • • •}

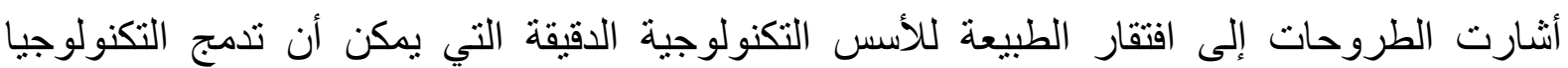

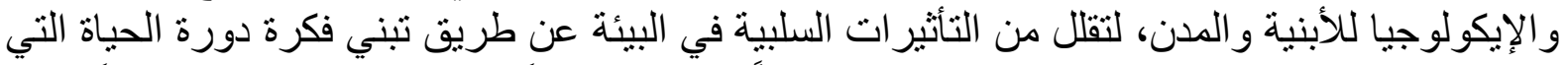

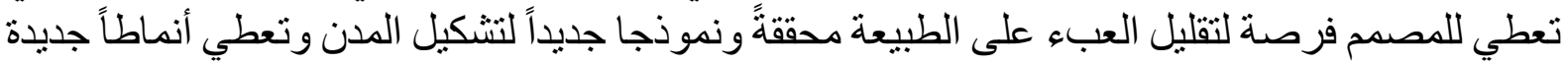

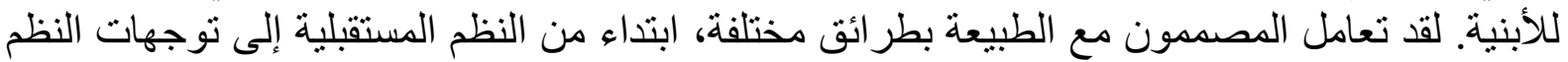

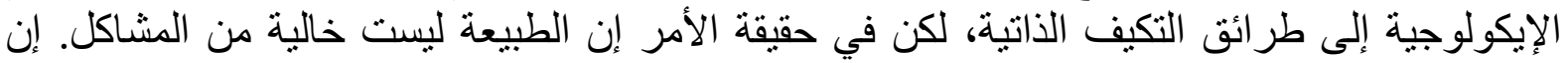

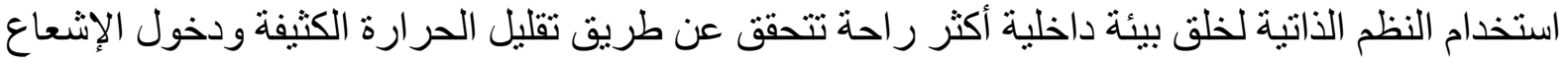

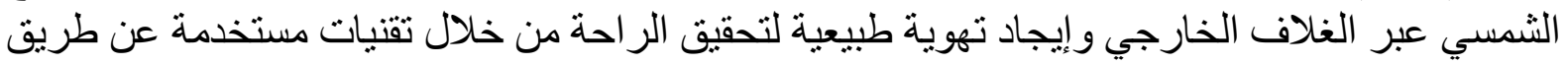

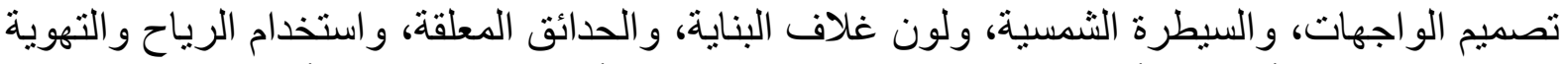

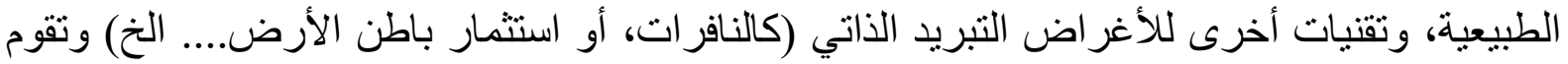

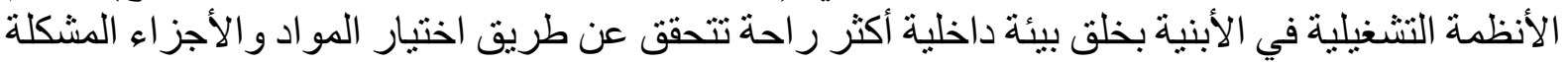

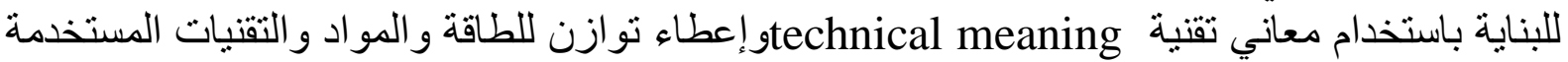

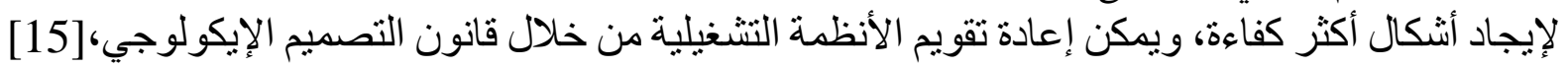

نستتتج مما سبق إمكانية تحقيق الاستدامة الحضرية من خلال إتباع بعض الاستر اتيجيات منها استخدام

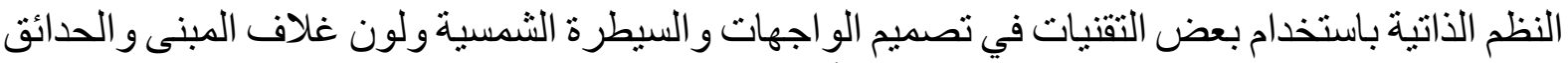

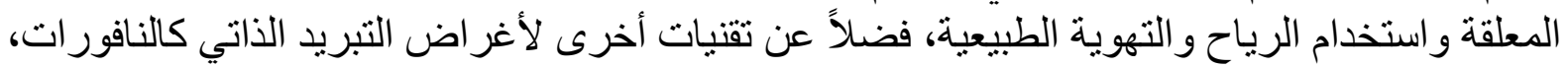

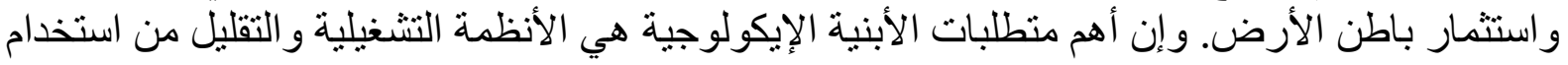

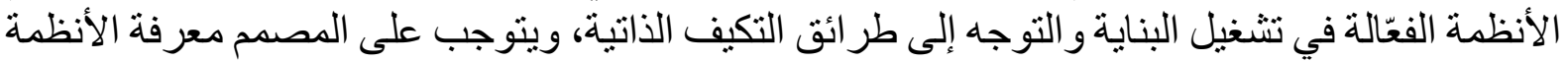

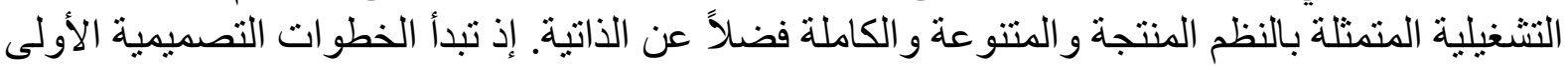

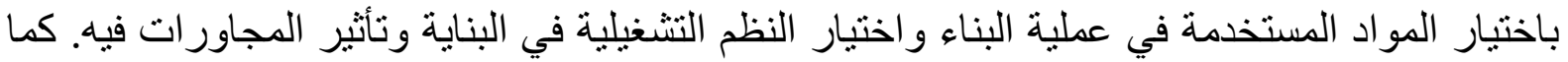
يمكن استتناج العلاقة الطردية بين زيادة الطاقة و التكنولوجيا للنظم التشغيلية.

\section{(Daniels, 1998) •}

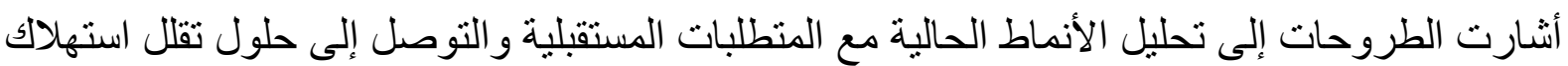

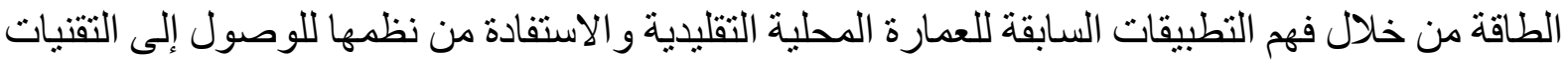

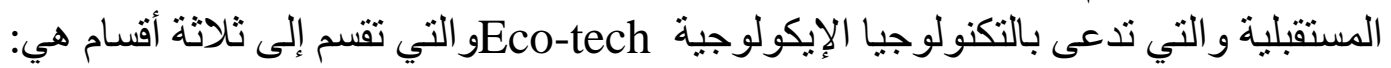
التقنية الو اطئة :- يمكن التوصل من خلال تحليل هذه التقنية الى انها التقنية البسيطة المتبعة في البيئة التقليدية المعتمدة على النظام المناخي. 
التقنية الخفيفة :- هي تقنيات بنائية أحدث من السابقة تتميز بأنها اخف وزناً ولها مرونة عالية، فمن خلال هذه المو اد يمكن الحصول على مبنى متكيف ذاتياً. التقنية العالية :- تعد من أفضل التقنيات المتاحة لتحقيق مبانٍ ذات التيات ادائية بيئية عالية، وتتميز التقنية

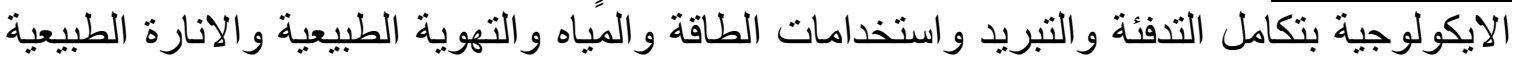

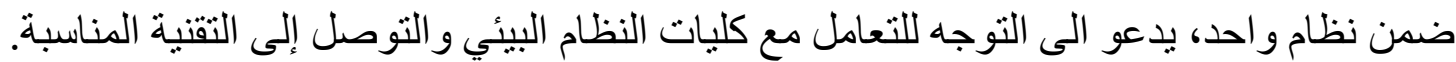

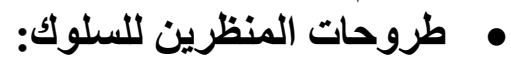

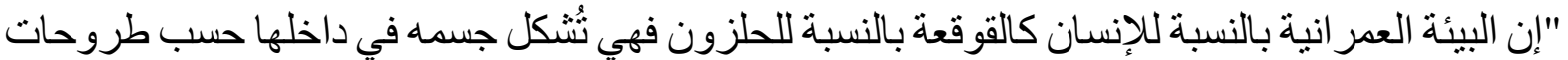

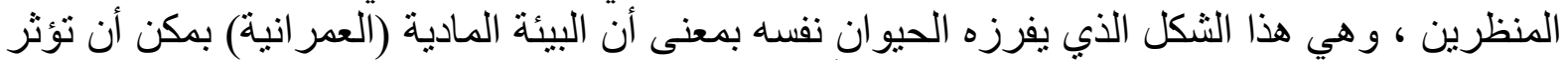

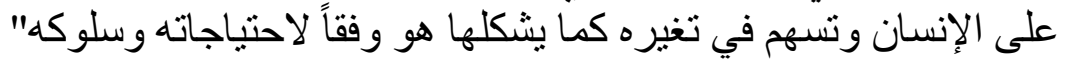

نظرية كورت لويين (Lewin) للسلوك: عبر لوين عن العلاقة بين سلوك الإنسان والبيئة المحبطة من

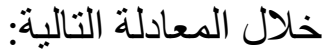

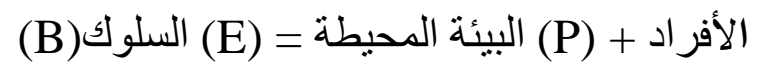

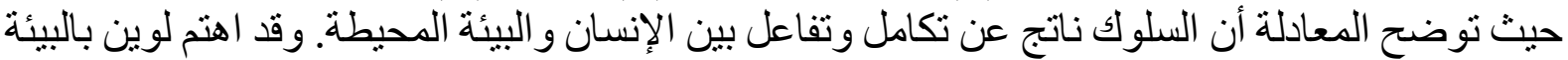

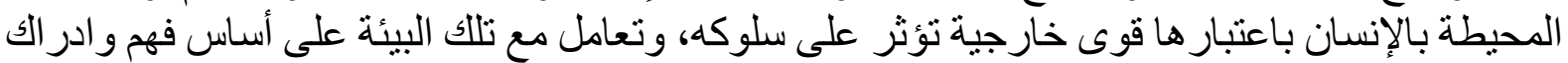

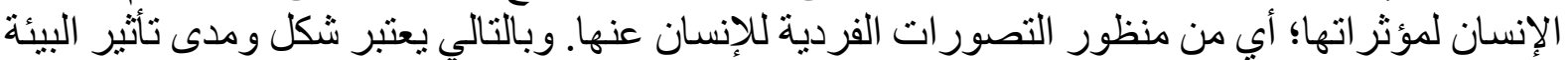

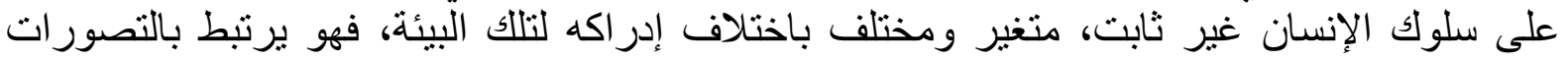

نظرية روجر باركر للمجال الســلوكي Behavior Setting Theory: قام كل من باركر Barker

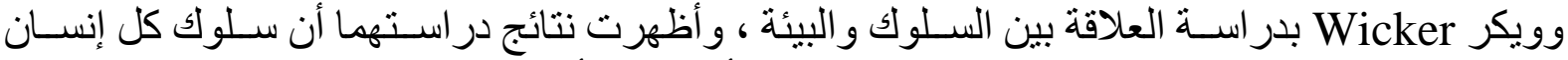

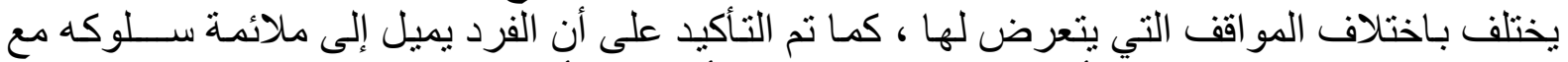

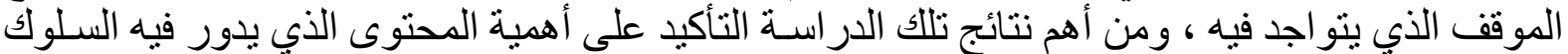

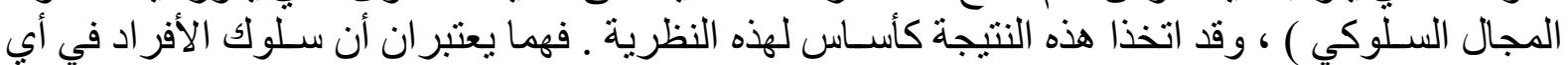

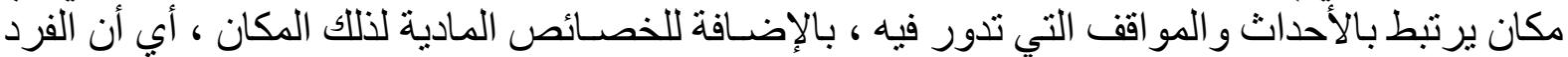

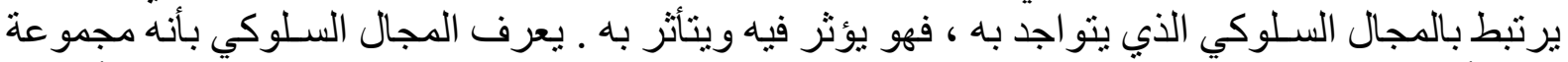

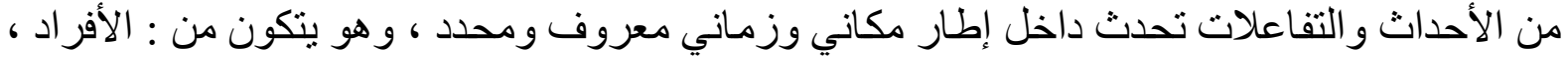

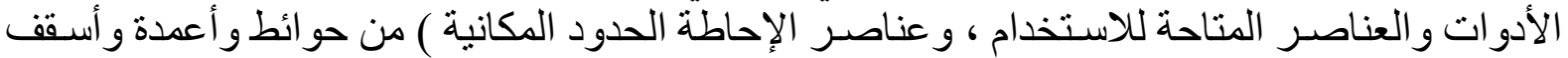

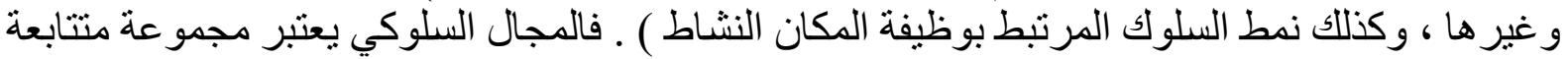
من الأحداث تحدث خلال زمن معين ، وتتحقق وحدته من خلال التو افق بين مكوناتـه ( بـاهر ، و خلود

\section{المواصفات التي يتم اعتبار ها عند تصميم المبنى بيئيا وايكلوجيا:}

مو اصفات داخل المبني : درجة الحر ارة التصميمية الداخلية في الثتاء و الصيف، و التهويةو الرطوبة النسبية المتوقعة .

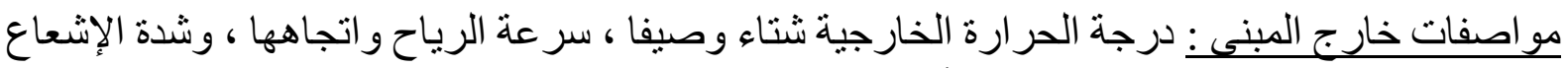

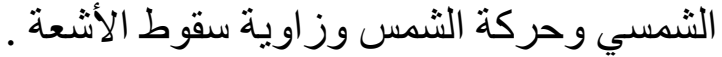

\section{مو اصفات الموقع الجغر افي :-}

قمم الجبال : أكثر عرضة للإشعاع الشمسي والرياح.] [16]

الثواطئ و السواحل: منوسطة التعرض للإشعاع الثمسي و والرياح حسب التوجيه 
الأر اضي على الهضاب أو التلال: متوسطة التعرض للإشعاع الثمسي والرياح حسب التوجيه

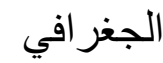

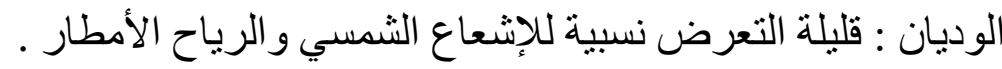

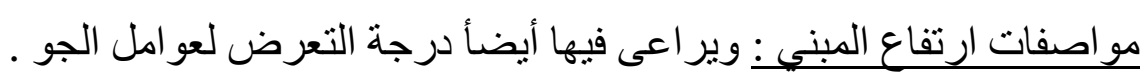

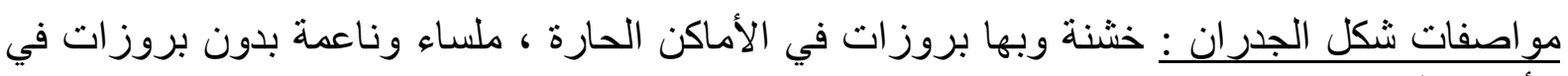

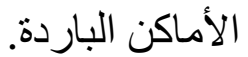
المصاعدات شيرهل المبنى : ــ المباني العالية تكون أقل تسريبا للطاقة ولكنها تحتاج إلى خدمات أكتر منل ـ المباني غير المرتفعة تكون أكثر تسريبه للطاقة ، لكن بها إضاءة نهاريةوتهوية

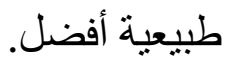
مو اصفات شكل سقف المبنى : ــ الأسقف المنحنية ، يقل معها مساحة الجزء المعرض لأشعة الثمس عند السطح . ـ القباب ، تحمي الجدار من الحرارة الز ائدة وخفض درجة حرارة السطح

مو اصفات الغلاف الخارجي للمبنى. مو اصفات المو اد المستخدمة في البناء : يلاحظ أن المباني في الحضار ات القديمة كانت تستعمل مواد بناء

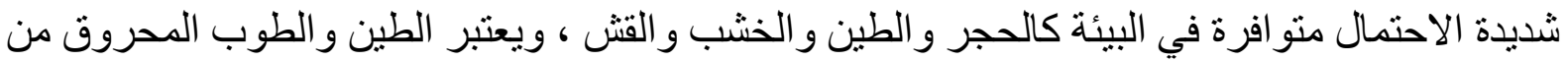

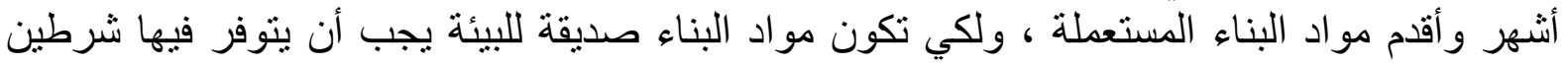
أساسين: ـ ألا تكون من المو اد عالية الاستهلاك الطاقة سواء في مرحلة التصنيع أو التركيب أو الصيانة .

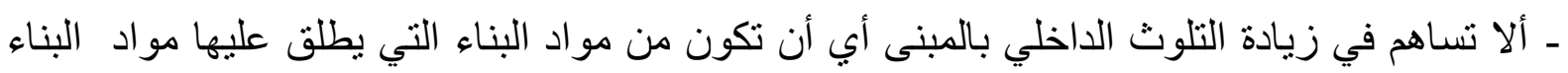

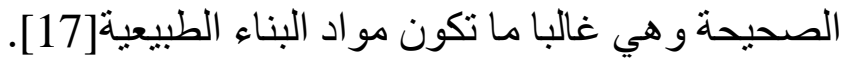

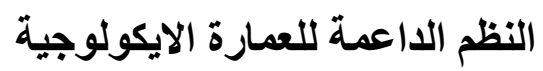

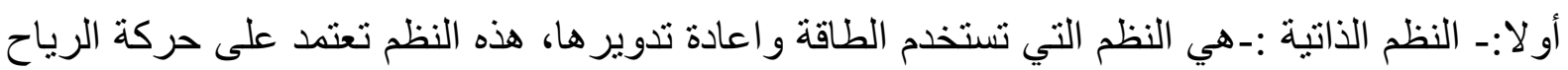

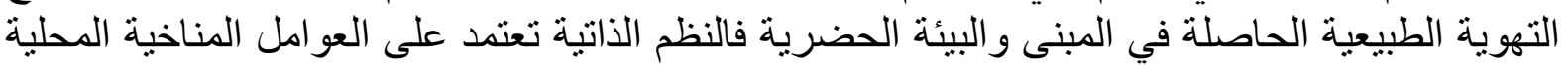
للموقع إذ تستخدم هذه النظم لأغر اض التبريد و التدفئة الذاتية من خلال الاعتماد على الطاقات الطبيعية.

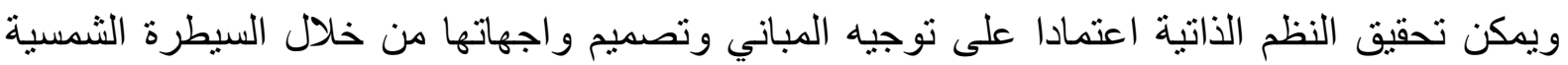

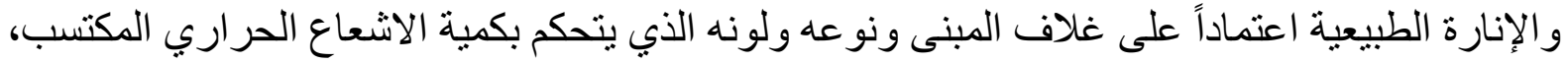

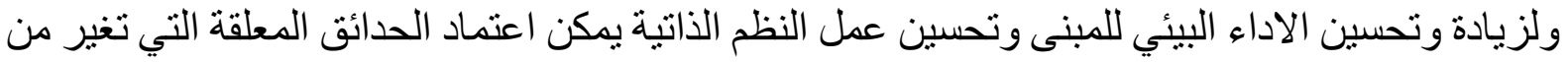

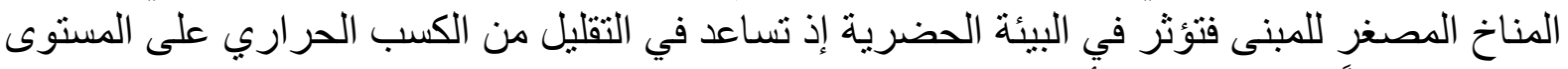
العمودي فضلاً عن المستوى الآفق الكن

أنواع النظم الذاتية:

1 التصميم الذاتي عن طريق تشكيل المباني 2. التصميم الذاتي عن طريق تصميم الواجهات :- 
يمكن ان تصمم هذه الواجهات عن طريق نظم حسابية خاصة لمعرفة مدى استجابتها للمؤثرات البيئية،

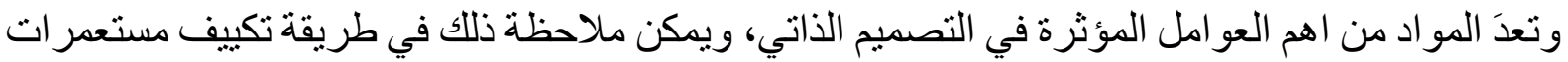
النمل الابيض للمحافظة على الاجواء الملائمة داخل المستعدرة ذات الجدار المزدوج لأغر اض الفئ التهوية

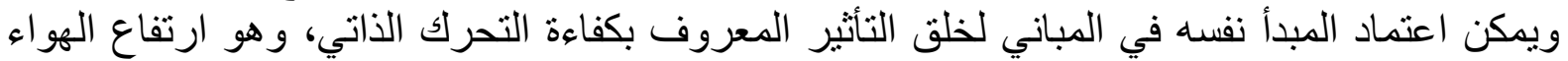

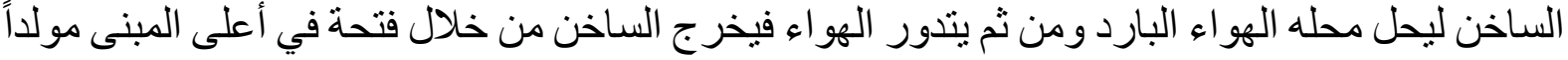

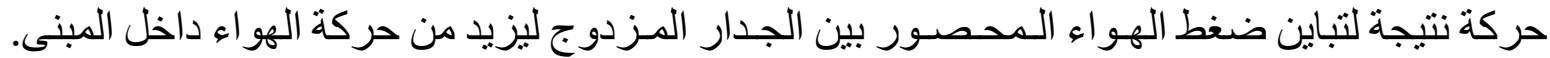

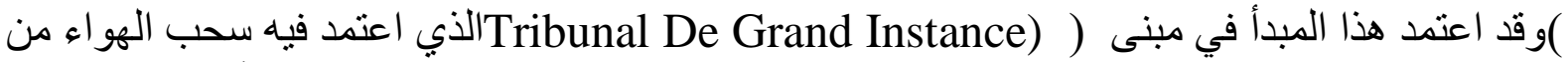
خارج المبنى عبر المسطحات المائية وادخاله الى المبنى ومن ثم تداور الهو اءو وتكيفه ذاتياً [18].

$$
\text { 4-التصميم الذاتي عن طريق لون غريق الذيطرة الثمسية }
$$

يدعو التصميم الذاتي إلى ظاهرة استخدام المواد ذات الألوان الفاتحة و لا سيما سقف المبنى الذي يكتسب

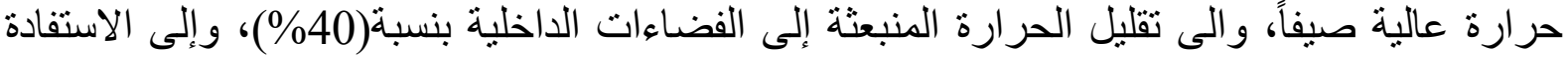
القصوى من التشجير حول الـمبنى للتقليل من الحرارة، وتدعى هذه الظاهرة بتأثنير الجزر الحرة الحرارية

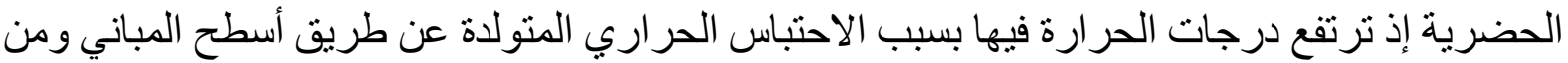

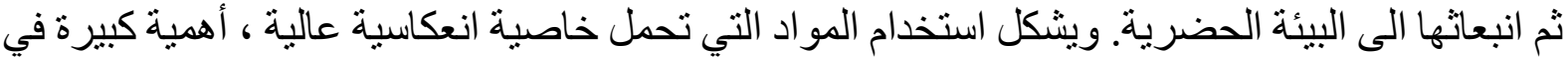

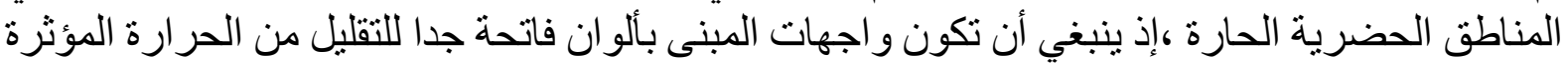
في الشوارع المحيطة بالمباني مؤدية إلى زيادة درجة حانية حر ارة البيئة الحضرية. 5. التصميم الذاتي عن طريق الحدائق المعلقة

6- التصميم الذاتي عن طريق استخدام الرياح و التهوية الطبيعية استخدام التقنيات البسيطة لأغر اض التبريد

الذاتي و هذه الطريقة هي احد طرق الاستدامة كما في مشروع (Century African House) حيث اعتمد المشروع فكرة المبنى النابع من البيئة من ناحية اعتماد الأشكال الطبيعية والانسجام معها و استخدام المواد المحلية لزيادة التناغم مع البيئة الطبيعية. يتسم المبنى بكونه ذا توجيه كفوء يحقق كفاءة

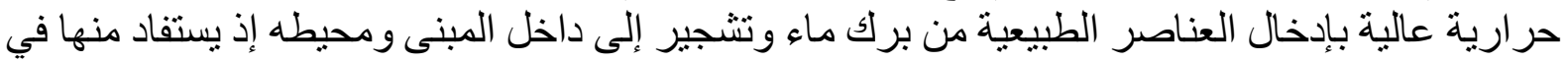
تحسين ادائية الأنظمة الذاتية المعتمدة على التهوية الطبيعية إذ يدخل الهو اء المبرد نتيجة لمروره فوق البرك

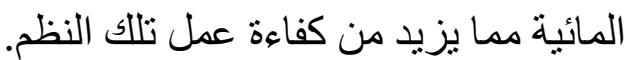
نستتج ان النظم الذاتية هي الأكثر فعالية للتصميم الإيكلوجيا كونها تتضمن كافة مكونات المباني وتعتمد

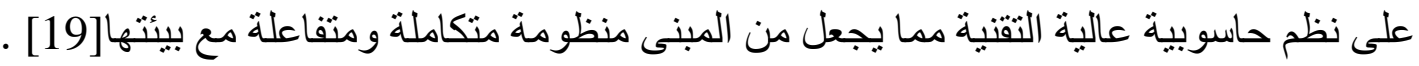

نظم الدوائر المفتوحة :- هي النظم التي تتحدد فيها الانبعاثات من النظام البيئي وقابلية النظام الايكولوجي على امتصاصها تعتمد فكرة النظام المفتوح المتماس مع البيئة على التحليل و على اساس تداخلات البيئة الطبيعية والمعمارية

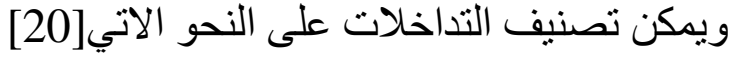
الاولى : العلاقة الخارجية، تتضمن النظم التصميمية و علاقاتها بالبيئة الخارجية. الثانية : العلاقة الداخلية، تتضمن النظم التصميمية و علاقاتها الداخلية. الثالثة : التحو لات الخارجية الى الداخلية، الطاقة و الموارد و أنظمة المدخلات 


$$
\text { الرابعة: التحو لات الداخلية الى الخارجية، تبادلات الطاقة و أنظمة النواتج }
$$

نظم الدوائر المغلقة المعنية بتحليل الانبعاثات الى مكونات ابسط ومن ثم تقليل التأثير في البيئة الحضرية و الطبيعية

نظم الدو ائر المغلقة المعنية بتحليل الانبعاثات الى مكونات ابسط ومن ثم تقليل التأثير في البيئة الحضرية و الطبيعية

النظم المتنوعة :- التي تمزج بين الأنظمة الفعالة و الذاتية. النظم الكاملة :- هي النظم التي يكون تأثير ها في البيئة قليلاً. • النظم المنتجة :- هي النظم التي تولد طاقة ذاتية ضمن حدود الموقع.

$$
\text { • النظم التكنولوجية و الرقمية }
$$

\section{التطبيق العملي \\ أبراج البحر في أببو ظبي}

يرهف تصميم هذا البرج إمكانية تحقُق الر احة الحر ارٌة بداخله وذللك من خلال إلقاء الضوء على على تكنولوجيا

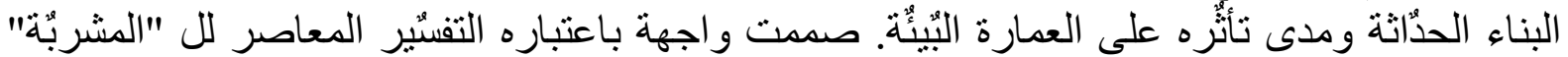

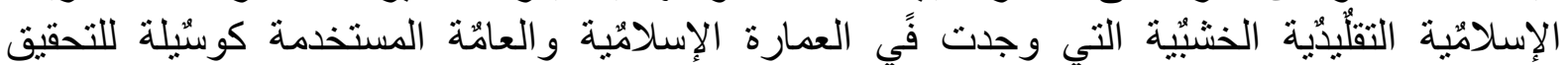

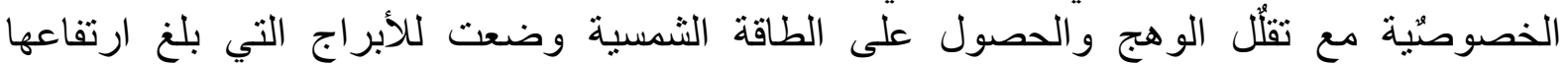

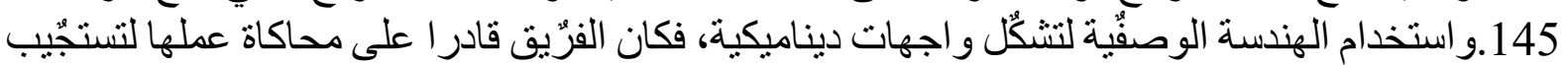

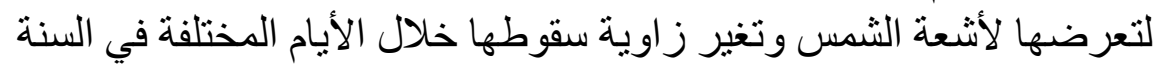
أهمية اختيار المشروع عُاعتبر رمزا هاما لترشُد الطاقة والربط بُين التكنولوجيا البناء و التصمُّيم البيئي ،حُيث الاستفادة لقصوى من الطاقة الثمسيّية والتحكم في الحر ارة الثمسئة المكتسبة ومقاو مة العو امل الثُيئية من رُّياح و شمس و حرارة.

مو اد البناء-: +يتكون سطح المبنى الخارجي من مضلة شبكية من الالياف الزجاجية بأطار من الالمنيوم

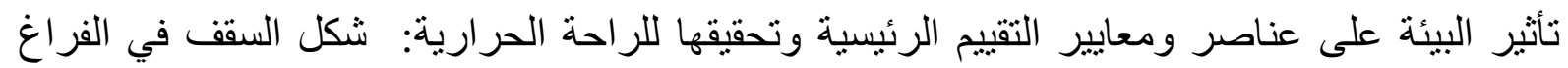
المعماري لقد اثرت فكرة ترشُد استهلاك الطاقة و التحكم البيئي على شكل الكتلة و السقف المائل للحماية

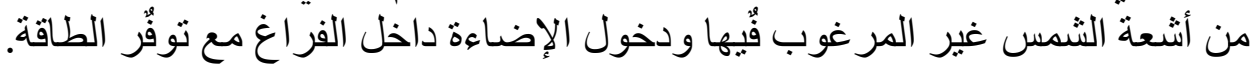

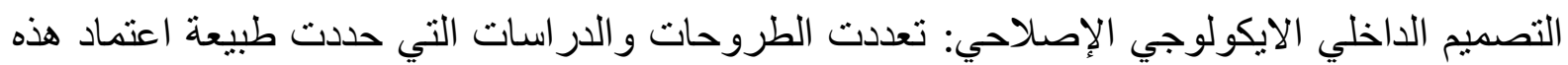
الاستر اتيجية في العمارة الداخلية ، اذ اشارت دراسة ( Kellert / 2004 ) الإهلى ان هناتك هدفان أساسيان

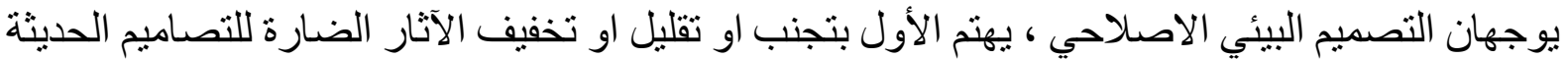

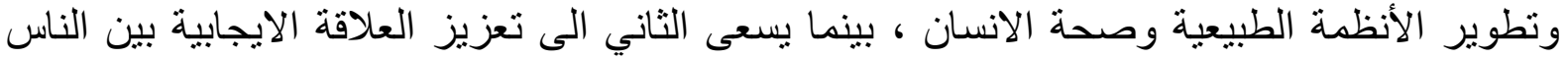

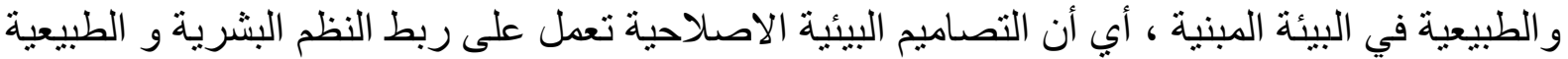

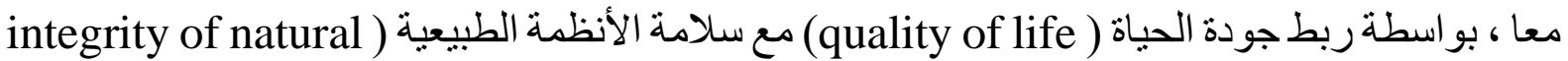
( systems

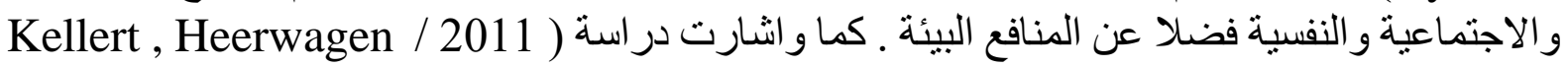
الرو \&ador الرو ابط الإيجابية بين الناس و الطبيعة في البيئة المبنية ضمن نموذج تصميم بيئي الصلاحي بحقق تصميم

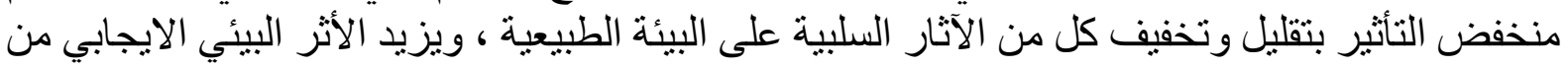


خلال كفاءة الطاقة وحفظ الموارد وتجنب التلوث وتقليل النفايات وذلك باستخدام منتجات ومو اد صديقة للبيئة وحماية جودة البيئية الداخلية . وبينت در اسة كل من ( 2011 / مugent , Packard , Brabon

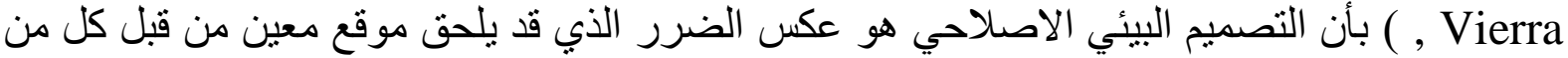
الطبيعة أو البشر ، حيث تعمل المباني المصممة على وفق التصميم البيئي الاصلاحي على على تحسين و اصلاحلح

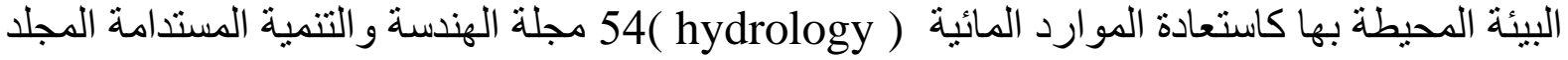

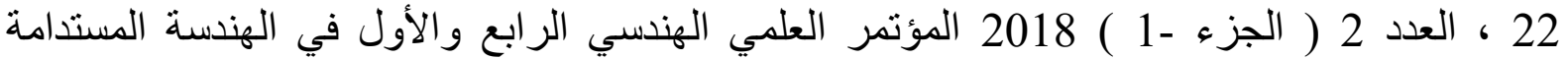
plant الطبيعية للموقع او توفير الحياة البرية المفقودة و المساكن النباتية (www.jeasd.org ( ISSN)

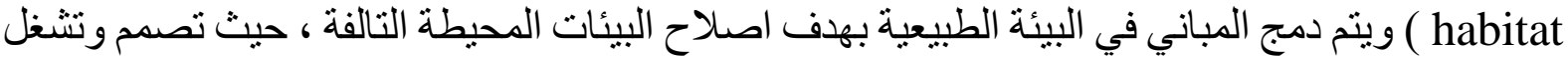

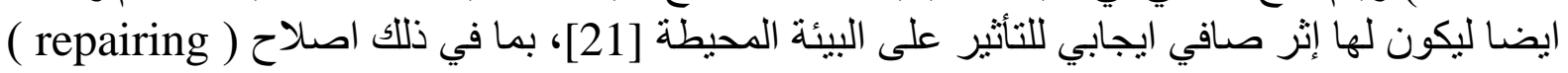

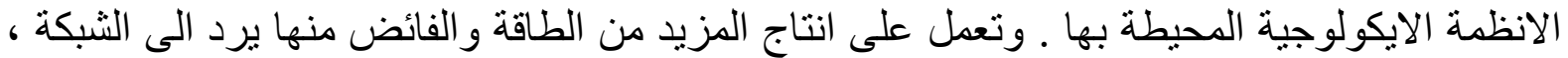

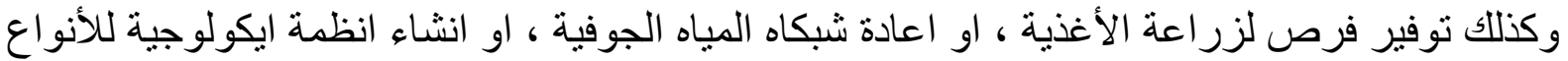

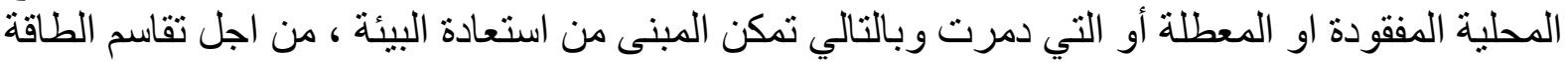
الز ائدة مع المباني المحيطة المنتجة وبالتالي توليد الطاقة في الموقع ويعتمد التصميم الإصلاحي على :

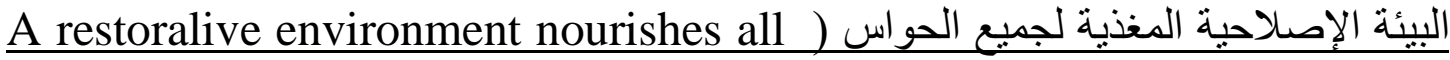
( the senses

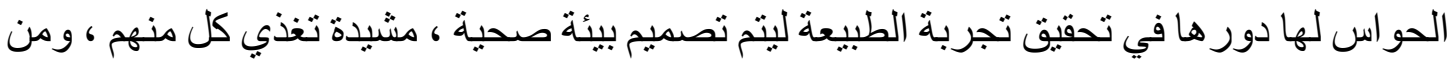

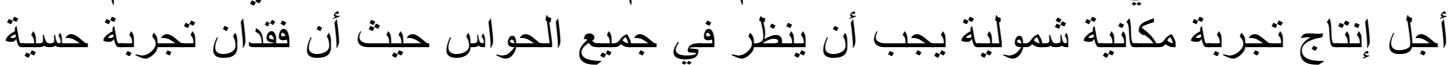

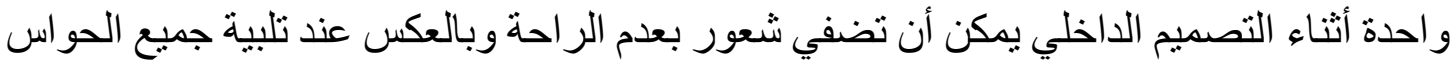

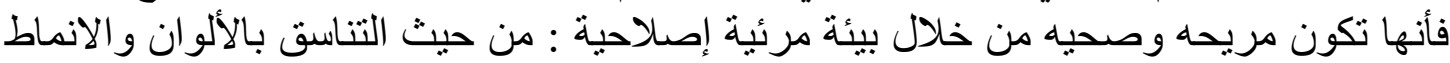
و الكتل ذات محفز ات إيجابية ، مثل الفن أو الطبيعة أو الموسيقى ( مشاهد طبيعية أوطبيعة مباشرة

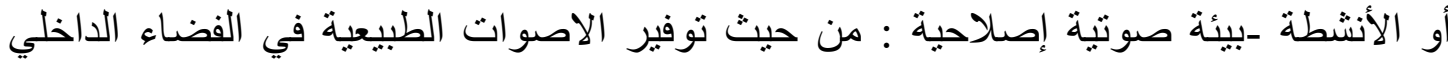

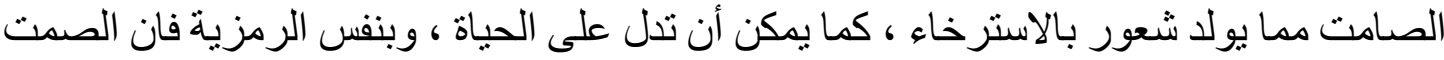

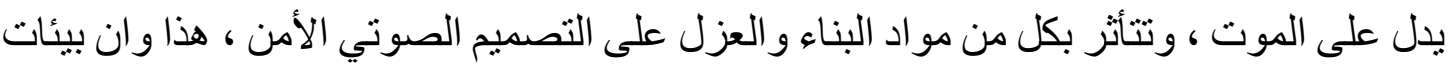
المكاتب المفتوحة توفر تقليل للضوضناء [22] ـ

المو اد ( materials ) : وتتحدد بالمو اد السطحية ل ( الأرضية ، السقف ، الجدران المستخدمة في

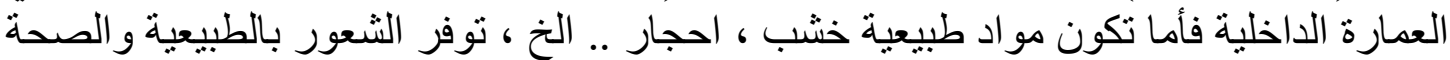

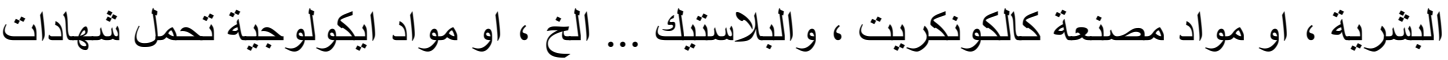

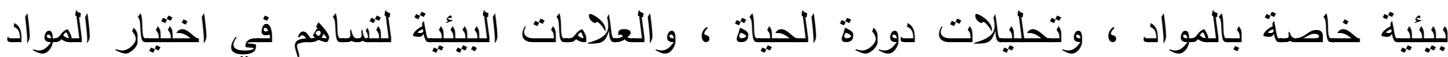

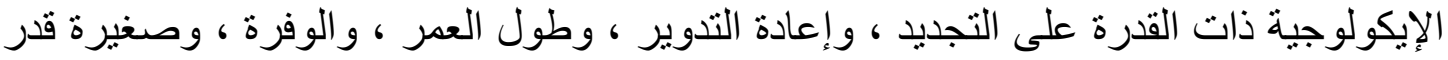

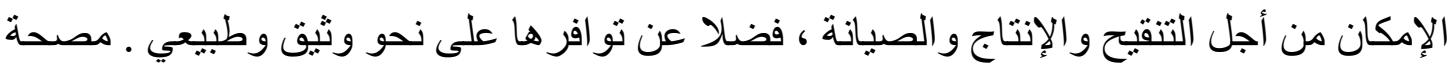

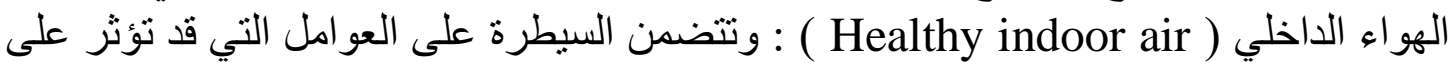

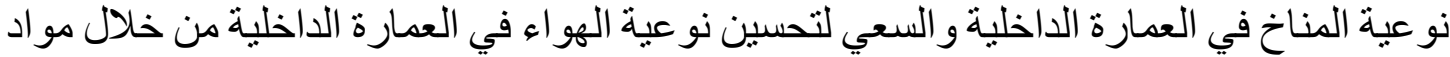
خالية من الانبعاثات ، و استخدام النباتات ، التهوية الطبيعية ، و استخدام فلاتر لتنقية الهو اءواء الداخلي

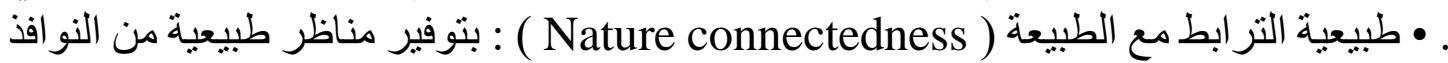

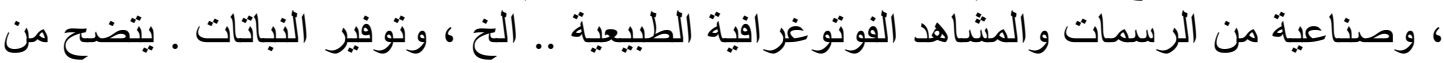

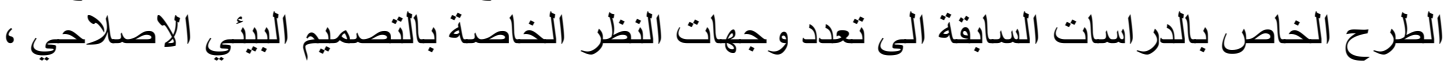

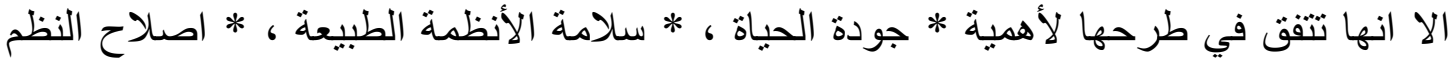

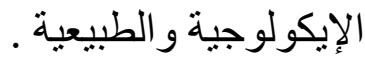


تطوير وتأهيل فضاء/ت مطعم كنتاكي هاوس ، كريلاء / 2017 لشركة الحجارة وفريقها التصميير ( تتضمن عملية التأهيل الخاصة بالمبنى ، انثاء جدار اخضر بمو اد وتقنيات محلية وذللك لمعالجة حالة

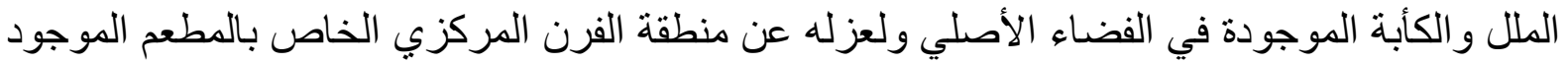

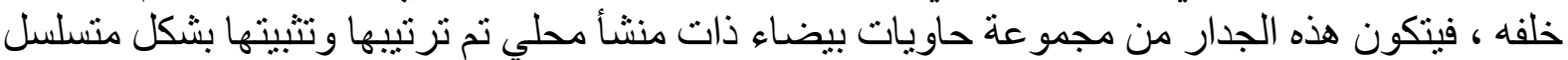

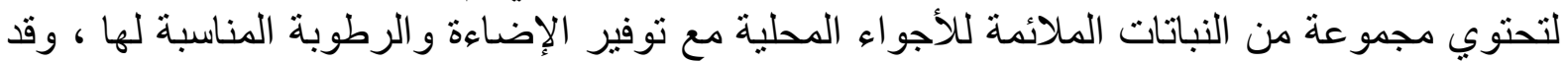

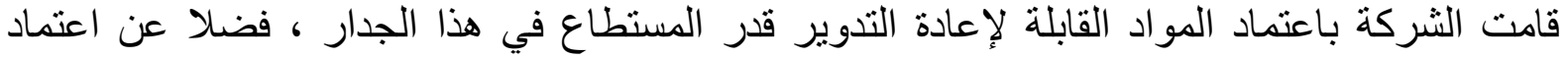

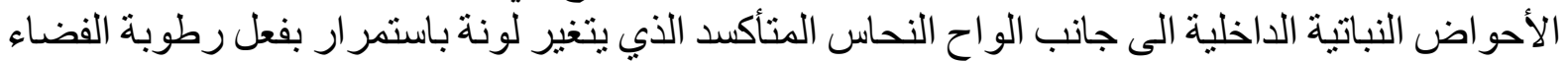

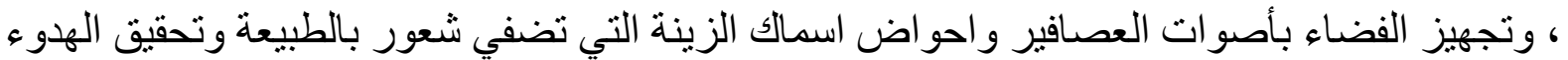

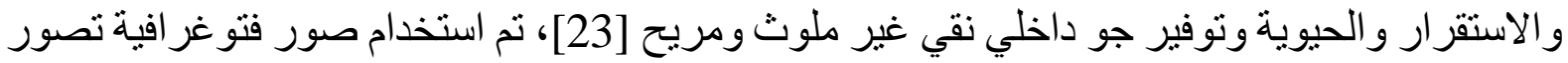

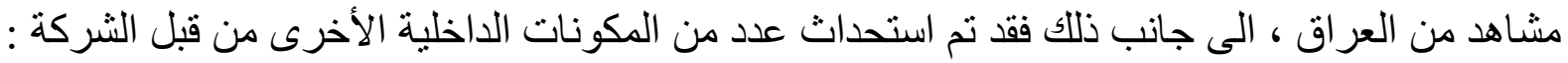

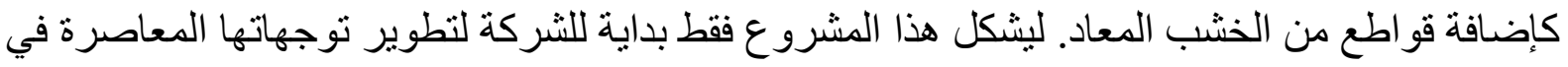

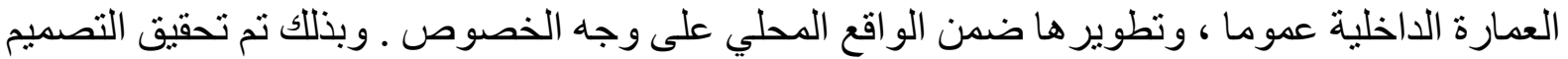
الايكولوجي للأنظمة الحية في العمارة الداخلية ، ومن خلال استر اتيجية التصميم الداخلي المحب التبل للطبيعة ،

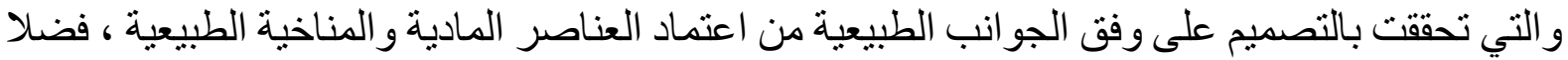

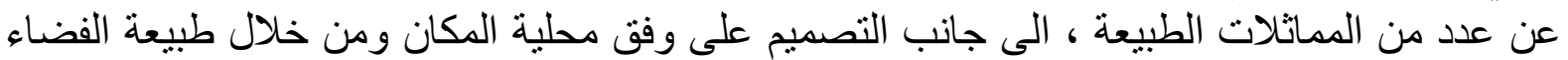

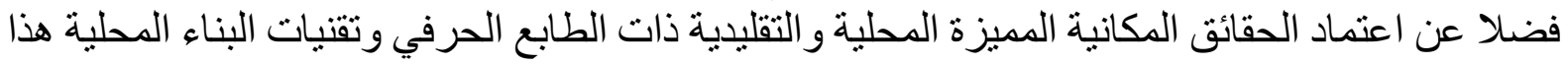

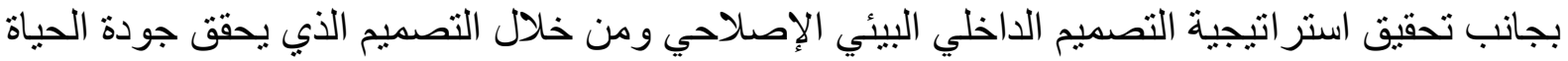

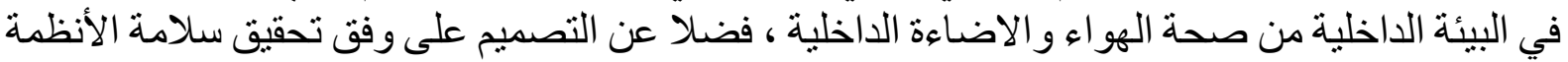

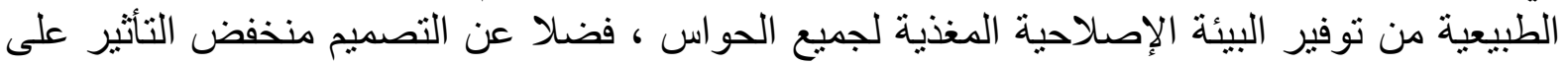

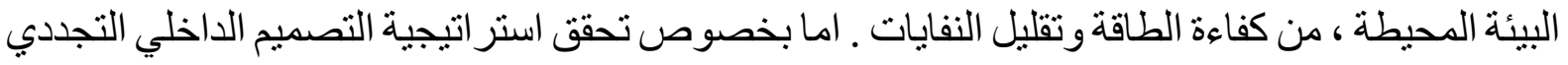

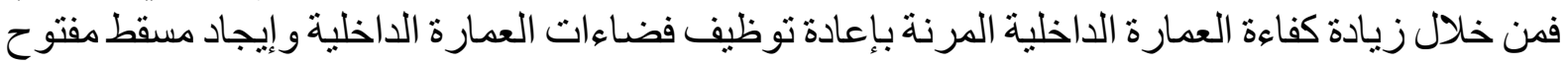

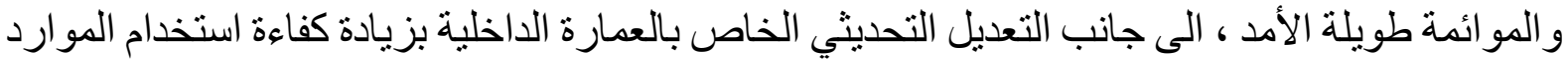

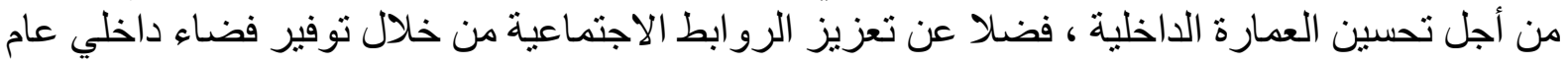

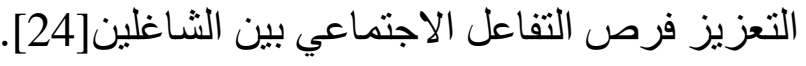

\section{(The Gherkin ) Str . Mary Axe in London 30 مبنح}

يعتبر أول وأطول مبني أيكولوجي في العالم ، فالشكل البيضاوي المشتق من شكل الخيار الصغير كما موضح في شكل ( 5 ) يعزز تدفق الرياح حول الجدران و الحد من الضغط علي الهيكل الذي سمح ايضا

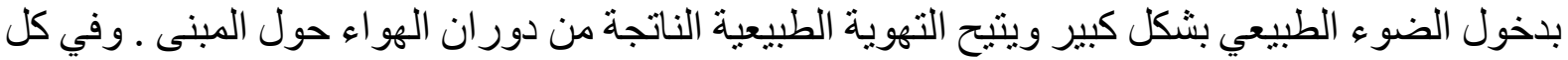

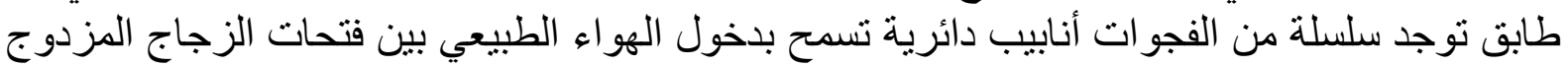
وتستخدم في التدفئة في الثتاء .

مما ادى للسيطرة على المناخ في هذا المبنى الي توفير في الطاقة الداخلية بنسبه اكثر من 50 \% ـ ـ ولقد تم

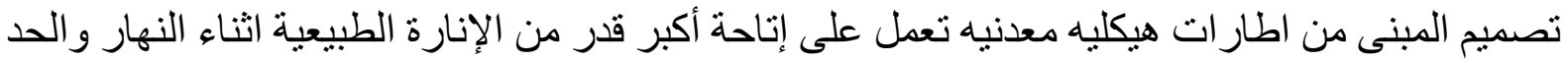

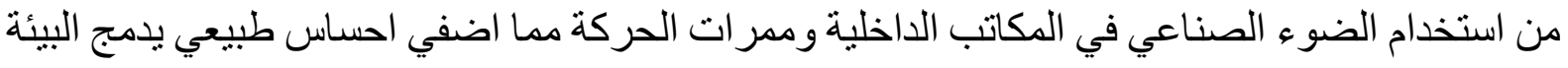
الخارجية و الداخلية معا [25].

ولقد لعبت المو اد الايكولوجية الحديثة ايضا دورا هاما في الحفاظ على استدامة المبنى وظيفيا وجماليا .

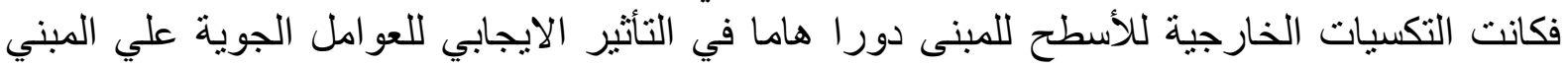

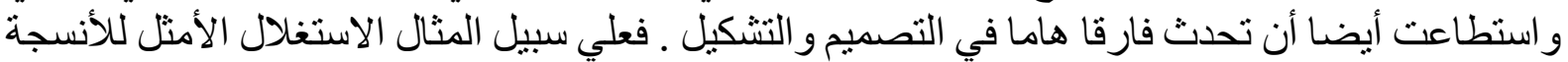

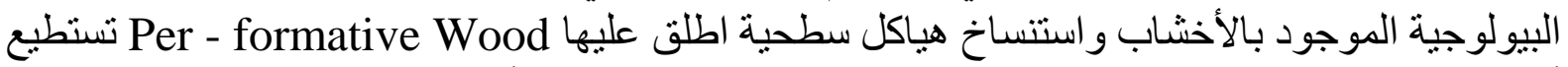
أن تمتص الرطوبة وتتعامل بشكل طبيعي مع التقلبات البيئية ساهم أيضا في تعويض النقص التص المتز ايد 
للأخشاب وفي تعزيز القدرة الوظيفية والثكلية وزياده القدرة الأدائية للمسطحات الخشبية وذلك عن طريق محاكاه شكل الألياف الطبيعية الموجودة بالخشب الطبيعي وتوظيف عمليات البرمجة الحسابية لكي تحاكي السلوك الطبيعي للأخشاب التطوير اسطح مساميه قادره على التكيف مع مستويات الرطوبة الطية.

\section{The New AUC Campus الحرم الجامعي الجديا للجامعة الامريكية بالقاهرة}

يعتبر المفهوم المعماري و البيئي التصميم مباني حرم الجامعة الامريكية بالقاهرة الجديدة نموذجا و اضحا

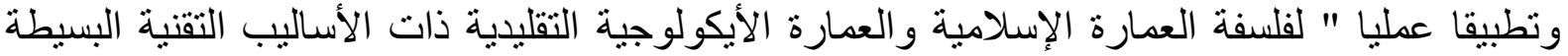

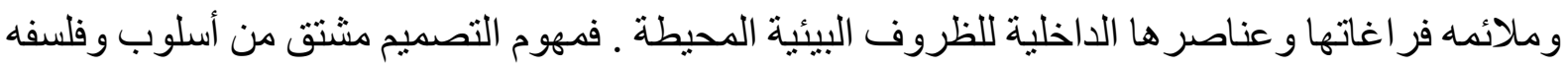
عماره العصر المملوكي. ولقد فرضت طبيعة الموقع والبيئة المحيطة الاعتماد على النظام البيئي الغير الغئي مباثر ( النظام الطبيعي ) ، [26]حيث يتم التحكم في العو امل المناخية الخارجية باستخدام الجدر ان السميكة للعزل الحر اري الداخلي و التظليل والنو افذ التي تنتهج نفس اسلوب المشربية في التبريد الداخلي ـ ولقد كان التهان

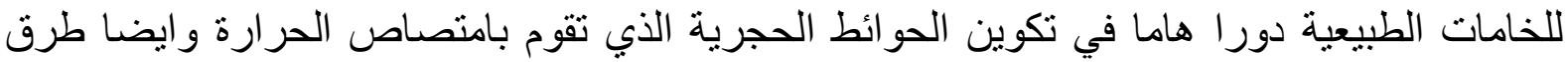

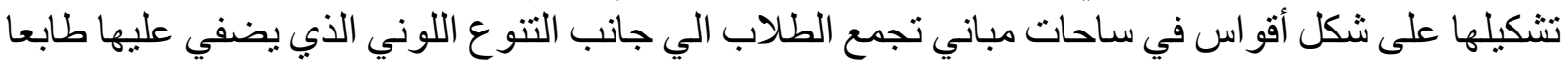

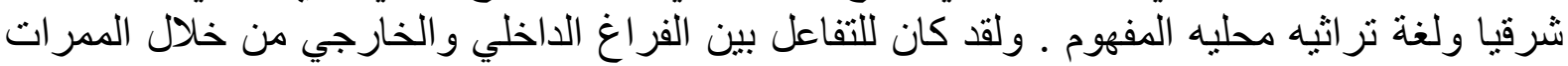

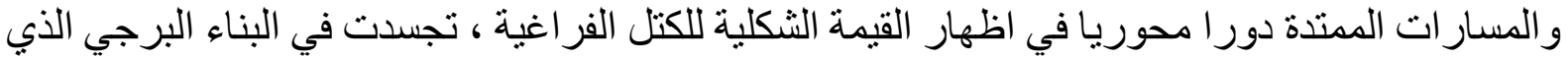

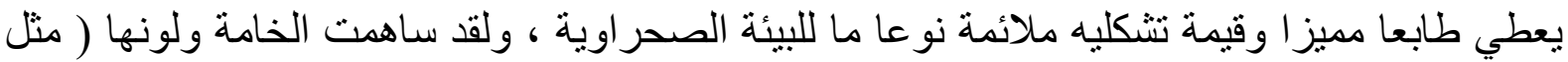
الحجر الرملي ) في إضفاء صفه ذاتيه طبيعية في الثكل و الوظيفة علي المساحات الداخلية و الخارجية

ويتضح التكامل بين معالجات التصميم الداخلي والخارجي في جميع أركان مباني الجامعة ، ولقد كانت

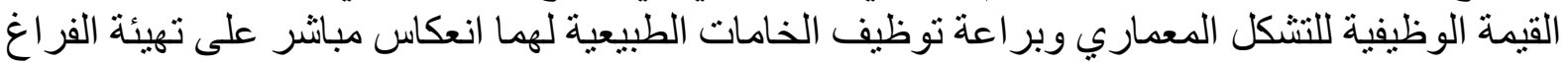

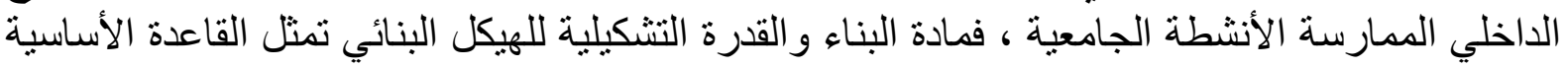

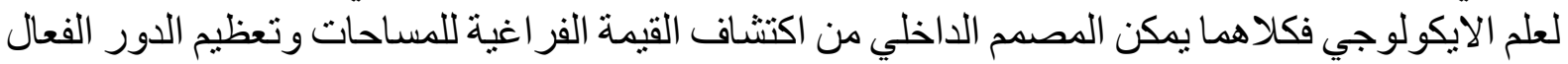

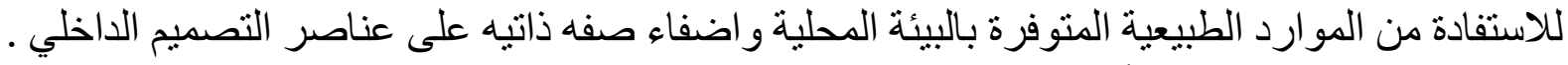
ولقد لعب الملمس دورا هاما أيضا في التعبير عن حيوية واندماج الفر اغات الفات الداخلية و الخارجية من خلال التفاعل بين الوحدات الإنشائية ودرجات السلالم المتكررة و الممتدة من الخارج إلى الفي الداخل و التي تم تكسيتها

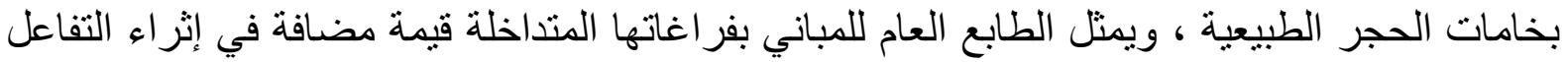

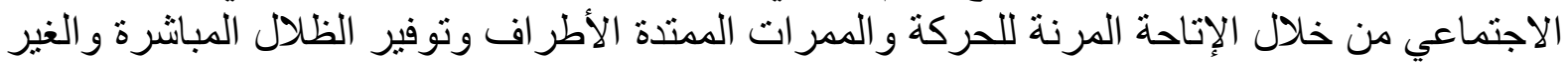

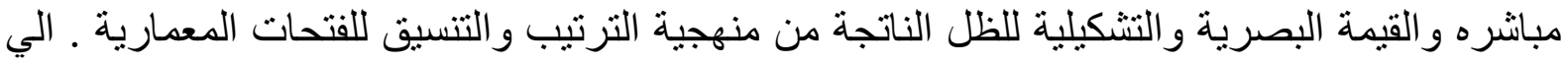

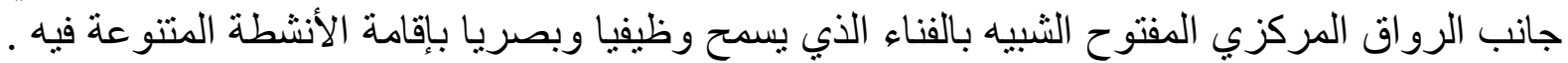

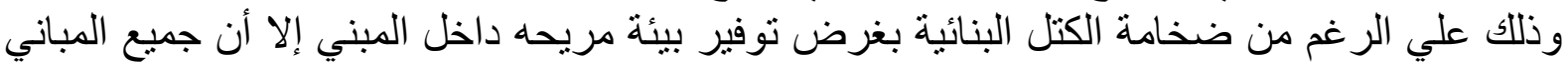
تميزت بوضوح الرؤية للفر اغات الداخلية والتي ظهرت مندمجة مع الطبيعة المحيطة [28].

الاستتناج

تعد البيئة المحيطة بالإنسان من اكثر العو امل المؤثرة على صحته وسلوكه.

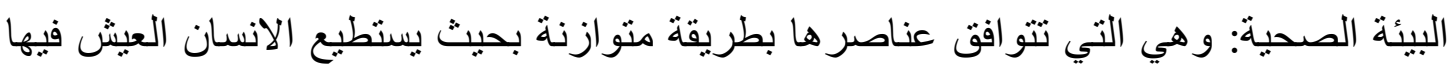

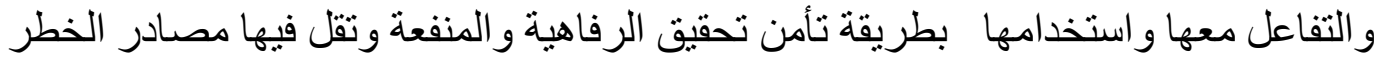

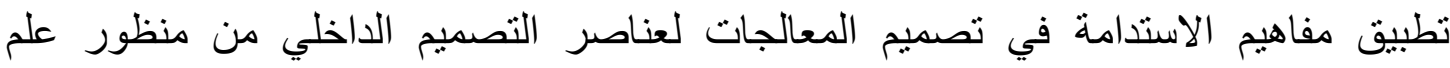

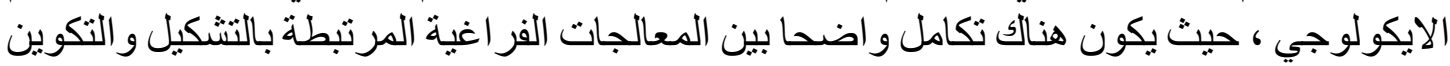

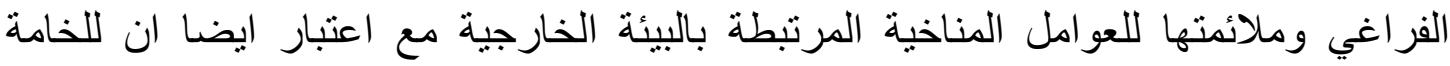

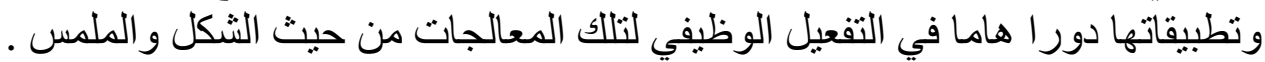


جميع خصائص البيئة المادية تؤثر على السلوك الإنساني بما يتضمن الظروف المناخية و الخصائص و المكونات التشكيلية للبيئة المبنية من أشكال و ألو ان و مساحات ولئية وحجوم و غير ها. إن العو امل السلوكية للإنسان تساهم بالإضـافة لعو امل أخرى في تشكيل إيكولوجيا الهنها المدينة (بيئتها)

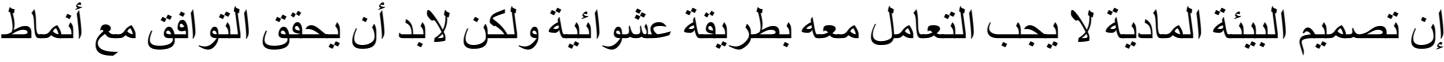
السلوك المر غوبة النة إن مجال علم النفس البيئي هو دراسة التفاعل بين الإنسان والبيئة المحيطة به، وهو بشمل البيئة الطبيعية و البيئة الصناعية علمئية (المثيّدة).

1. U. Schimmack, J. Schupp, and G. G. Wagner, "The influence of environment and personality on the affective and cognitive component of subjective well-being," Soc. Indic. Res., vol. 89, no. 1, pp. 41-60, 2008.

2. D. Garlaschelli, S. E. Ahnert, T. Fink, and G. Caldarelli, "Low-temperature behaviour of social and economic networks," Entropy, vol. 15, no. 8, pp. 3148-3169, 2013.

3. F. N. Freeman, K. J. Holzinger, and B. C. Mitchell, "The influence of environment on the intelligence, school achievement, and conduct of foster children.," Yearb. Natl. Soc. Study Educ., 1928.

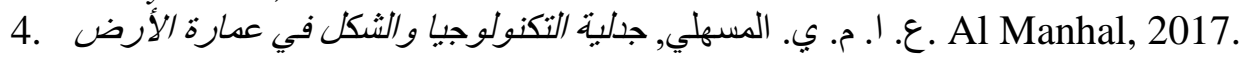

5. L. R. Good, "Architectural Environment and Human Behavior,” Kansas J. Sociol., vol. 1, no. 2, pp. 56-60, 1965.

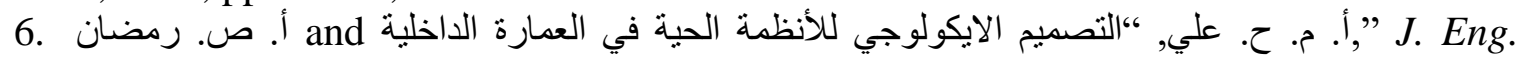
Sustain. Dev., vol. 22, no. 2 (Part-1), 2018.

7. D. T. L. Jordan, "Human Behavior and the Social Environment I," 2000.

8. S. L. Farhan, V. S. Akef, and Z. Nasar, "Revitalizing the historical center of Al-Najaf city in Iraq: learning from the British conservation experiences," J. Cult. Herit. Manag. Sustain. Dev., vol. ahead-of-p, no. ahead-of-print, Jan. 2021.

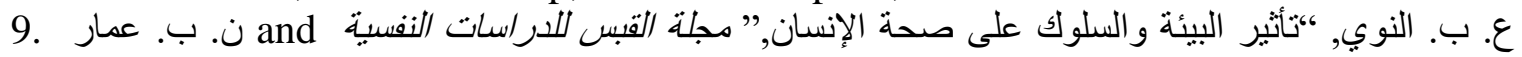
والإجتماعية, vol. 2, no. 6, pp. 86-103, 2020.

10. H. S. O. Adelphi, S. L. Farhan, and H. A. H. Alshamari, "The Threshold of Urban Sustainability within the Traditional Cities: Traditional Alnajaf city as a case study," IOP Conf. Ser. Mater. Sci. Eng., vol. 1058, no. 1, p. 12055, Feb. 2021.

11. M. Pantic, A. Pentland, A. Nijholt, and T. S. Huang, "Human computing and machine understanding of human behavior: A survey," in Artifical intelligence for human computing, Springer, 2007, pp. 47-71.

12. A. N. Sabeeh Lafta Farhan, Ihsan Abbass Jasim, "Urban sustainability in Old City Centres, a Comparison Between the City of Najaf in Iraq and Italian Cities Experiences," no. 12, 2016.

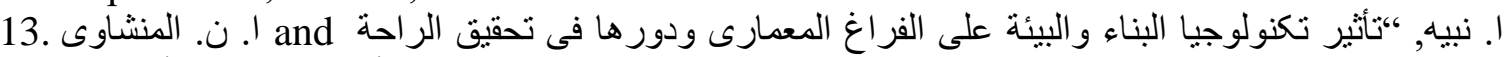

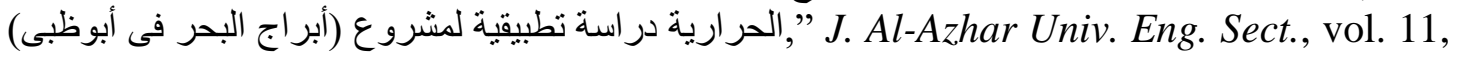
no. 38, pp. 343-359, 2016.

14. I. A. Jasim, S. L. Farhan, and H. M. Hasan, "Ways to Activate Urban Transport to Achieve Urban Sustainability," IOP Conf. Ser. Mater. Sci. Eng., vol. 1090, no. 1, p. 012034, 2021.

15. S. L. Farhan, I. A. J. Hashim, and A. A. Naji, "The sustainable house: Comparative analysis of houses in Al Kut neighborhoods-Iraq," Proc. - Int. Conf. Dev. eSystems Eng. DeSE, vol. October-20, pp. 1031-1036, 2019.

16. I. Abbass Jasim, S. Lafta Farhan, and S. AL-MAMOORI, "Smart Government: Analysis of Shift Methods in Municipal Services Delivery: The Study Area: Al-Kut Iraq," J. Univ. Kerbala, vol. 15, no. 3, p. 2017, 2017. 
17. S. Farhan and M. G. Abdelmonem, "Navigating the socio-spatial and planning conditions of traditional public spaces in Iraq's holy cities," in Traditional Dwellings and Settlements Review, 2018, vol. 30, no. 1, p. 81.

18. H. A. et al. S. Farhan, T.Mutas, "Digital Era Influence on Neighbourhood Planning," I O P Conf. Sci. Mater., 2021.

19. S. L. Farhan, "Sustainable neighborhood Comparative Analysis of Al Kut Neighborhoods Sustainable neighborhood Comparative Analysis of Al Kut Neighborhoods," no. December, pp. 302-317, 2018.

20. S. L. Farhan, H. H. Samir, and H. S. Adelphi, "Urban changes and its impact on the tangible and intangible heritage of City's Centre: Najaf City as a Case Study," IOP Conf. Ser. Mater. Sci. Eng., vol. 1058, no. 1, p. 12070, 2021.

21. S. L. Farhan, H. I. Alyasari, H. H. Samir, S. L. Zubaidi, and K. S. Hashim, "Conservation Approach as an Architectural Instrument to reviving Historical Cities; technical analysis for multi international cases," IOP Conf. Ser. Mater. Sci. Eng., vol. 1058, no. 1, p. 12071, 2021.

22. Z. A.; Farhan, Sabeeh Lafta 1; Nasar, "Urban identity in the holy cities of Iraq: Analysis trends of architectural designers in the city of Karbala," J. Urban Regen. Renew., vol. 14 Number, no. 2020, pp. 210-222(13), 2020.

23. F. Soflaee and M. Shokouhian, "Natural cooling systems in sustainable traditional architecture of Iran," Int. Conf. "Passive Low Energy Cool. Built Environ. Santorini, Greece, no. May, pp. 715-719, 2005.

24. H. H. Alzoubi and A. T. Almalkawi, "A comparative study for the traditional and modern houses in terms of thermal comfort and energy consumption in Umm Qais city, Jordan," J. Ecol. Eng., vol. 20, no. 5, pp. 14-22, 2019.

25. S. L. Farhan, H. I. Alyasari, V. S. Akef, S. L. Zubaidi, and K. S. Hashim, "Analysing the Transformed Urban Patterns of Al-Najaf Historical Center: Urgent Issues and Possible Solutions," IOP Conf. Ser. Mater. Sci. Eng., vol. 1058, no. 1, p. 12052, 2021.

26. N. B. Grimm et al., "Global change and the ecology of cities," Science (80-. )., vol. 319, no. 5864, pp. 756-760, Feb. 2008.

27. Sabih 1. Farhan, "Visual pollution in the commercial urban scene Analysis of the optical axes elected in the Kut city.," Wasit J. Eng. Sci., no. 4, pp. 178-208, 2016. 